\title{
Spontaneous functional network dynamics and associated structural substrates in the human brain
}

\author{
Xuhong Liao ${ }^{1,2+}$, Lin Yuan ${ }^{1+}$, Tengda Zhao ${ }^{1}$, Zhengjia Dai ${ }^{1}$, Ni Shu ${ }^{1}$, Mingrui Xia ${ }^{1}$, \\ Yihong Yang ${ }^{3}$, Alan Evans ${ }^{4}$ and Yong $\mathrm{He}^{1 *}$ \\ ${ }^{1}$ State Key Laboratory of Cognitive Neuroscience and Learning and IDG/McGovern Institute for Brain Research, Beijing \\ Normal University, Beijing, China, ${ }^{2}$ Center for Cognition and Brain Disorders, Hangzhou Normal University, Hangzhou, China, \\ ${ }^{3}$ Neuroimaging Research Branch, National Institute on Drug Abuse, National Institutes of Health, Baltimore, MD, USA, \\ ${ }^{4}$ McConnell Brain Imaging Centre, Montreal Neurological Institute, McGill University, Montreal, QC, Canada
}

\section{OPEN ACCESS}

Edited by:

Joshua Oon Soo Goh,

National Taiwan University, Taiwan

Reviewed by:

Betty M. Tijms,

VU University Medical Center,

Netherlands

Tsung-Ren Huang,

Boston University, USA

*Correspondence:

Yong $\mathrm{He}$

State Key Laboratory of Cognitive Neuroscience and Learning and IDG/McGovern Institute for Brain Research, Beijing Normal University, Beijing 100875, China yong.he@bnu.edu.cn

${ }^{\dagger}$ These authors have contributed equally to this work.

Received: 24 June 2015

Accepted: 17 August 2015

Published: 04 September 2015

Citation:

Liao X, Yuan L, Zhao T, Dai Z, Shu N, Xia M, Yang Y, Evans A and $\mathrm{He} Y$

(2015) Spontaneous functional network dynamics and associated structural substrates in the human brain. Front. Hum. Neurosci. 9:478.

doi: 10.3389/fnhum.2015.00478
Recent imaging connectomics studies have demonstrated that the spontaneous human brain functional networks derived from resting-state functional MRI (R-fMRI) include many non-trivial topological properties, such as highly efficient small-world architecture and densely connected hub regions. However, very little is known about dynamic functional connectivity (D-FC) patterns of spontaneous human brain networks during rest and about how these spontaneous brain dynamics are constrained by the underlying structural connectivity. Here, we combined sub-second multiband R-fMRI data with graph-theoretical approaches to comprehensively investigate the dynamic characteristics of the topological organization of human whole-brain functional networks, and then employed diffusion imaging data in the same participants to further explore the associated structural substrates. At the connection level, we found that human whole-brain D-FC patterns spontaneously fluctuated over time, while homotopic D-FC exhibited high connectivity strength and low temporal variability. At the network level, dynamic functional networks exhibited time-varying but evident small-world and assortativity architecture, with several regions (e.g., insula, sensorimotor cortex and medial prefrontal cortex) emerging as functionally persistent hubs (i.e., highly connected regions) while possessing large temporal variability in their degree centrality. Finally, the temporal characteristics (i.e., strength and variability) of the connectional and nodal properties of the dynamic brain networks were significantly associated with their structural counterparts. Collectively, we demonstrate the economical, efficient, and flexible characteristics of dynamic functional coordination in large-scale human brain networks during rest, and highlight their relationship with underlying structural connectivity, which deepens our understandings of spontaneous brain network dynamics in humans.

Keywords: connectomics, functional dynamics, graph theory, hub, small-world, sliding window

\section{Introduction}

Over the past two decades, resting-state functional magnetic resonance imaging (R-fMRI), a promising functional imaging technique, has been widely used to non-invasively map the brain's intrinsic or spontaneous functional connectivity patterns (i.e., functional connectome) by measuring the correlations in blood oxygen level-dependent (BOLD) signals between regions 
during rest (Biswal et al., 1995; for reviews, see Fox and Raichle, 2007; Kelly et al., 2012). Recently, the combination of R-fMRI and graph-based network analyses has allowed us to quantitatively characterize the topological characteristics of functional networks in the human brain, such as smallworldness, modularity, and highly connected hub regions (for reviews, see Bullmore and Sporns, 2009; He and Evans, 2010; Bullmore and Sporns, 2012). These studies provide mechanistic insights into the functional organization principles of human brain networks in health and disease.

To date, the majority of R-fMRI studies on functional connectomics have focused mainly on static functional connectivity (S-FC) by computing inter-regional statistical associations in whole-scan time courses, implicitly neglecting the potential temporal fluctuations of functional interactions across time. Recently, emerging evidence suggests that functional coupling among regions is highly dynamic at a time scale of seconds to minutes (Chang and Glover, 2010; Kang et al., 2011; Kiviniemi et al., 2011; Hutchison et al., 2013b; Allen et al., 2014; Gonzalez-Castillo et al., 2014; Di and Biswal, 2015). The dynamic functional connectivity (D-FC) can capture the transition between different mental states (Allen et al., 2014) associated with changes in electroencephalograph (EEG) power (Tagliazucchi et al., 2012b; Chang et al., 2013), and provide novel insights into the pathophysiological mechanisms of neuropsychiatric disorders, such as Alzheimer's disease (Jones et al., 2012) and post-traumatic stress disorder (Li et al., 2014). Specifically, several recent R-fMRI studies have further demonstrated the dynamic characteristics of the topological organization of human brain networks such as fluctuating modular architecture (Jones et al., 2012; Allen et al., 2014) and sporadic intervals of high network efficiency (Zalesky et al., 2014; Di and Biswal, 2015). As mentioned above, the brain's functional networks include many non-trivial topological properties, such as small-worldness, which quantifies an optimal balance between information segregation and integration (Salvador et al., 2005; Bassett and Bullmore, 2006), and highly connected hubs, which play key roles in global brain communication (Achard et al., 2006; Buckner et al., 2009; Liao et al., 2013; van den Heuvel and Sporns, 2013). However, how these crucial topological properties of human brain networks vary spontaneously over time remains largely unknown.

A growing number of neuroimaging studies have suggested that the human brain's functional organization is sculpted by the underlying anatomical structure (see Reviews, Park and Friston, 2013; Wang et al., 2015). At the connection level, two regions often exhibit high temporal synchronization if they are directly linked by white matter tracts (Greicius et al., 2009; Honey et al., 2009; van den Heuvel et al., 2009). At the network level, the brain's functional and structural networks share topological organization, such as small-worldness and hubs (Bullmore and Sporns, 2009, 2012). Notably, all of these previous studies have been mainly confined to the exploration of the structural basis of static functional connectivity or networks. Since the anatomical structure constrains the propensity of inter-regional interactions, it's natural to expect that ongoing brain activities and the accompanying dynamic functional coordination may be shaped by the underlying structural organization (Deco et al., 2013). Until recently, Shen et al. (2015) have demonstrated the structural-dependence of the spontaneous dynamic functional coordination in macaques at both the local and global levels. However, for the human brain, very little is known about whether and how the dynamic connectivity patterns of functional networks are constrained by the underlying white matter tracts or structural connectivity (SC).

To address these issues, in the present study we employed BOLD R-fMRI data and graph theoretical approaches to systematically characterize the dynamic topological properties of human whole-brain functional networks. Further, we used diffusion tensor imaging (DTI) data to construct white matter structural networks in the same participants to reveal the structural substrates underlying these functional network dynamics. Specifically, to better track the brain network dynamics at a fine time scale, a multiband R-fMRI dataset with a sub-second sampling rate $(\mathrm{TR}=645 \mathrm{~ms})$ was used here, which provides additional temporal information regarding BOLD signal activities. Here, we sought to determine (i) how the topological organization of intrinsic or spontaneous functional brain networks changes over time at different levels (including connectional, global and nodal properties); and (ii) how the white matter structural connectivity underlies these dynamic network characteristics.

\section{Materials and Methods}

\section{Participants}

The multiband R-fMRI and DTI data were selected from a publicly available dataset (http://fcon 1000.projects.nitrc.org/indi/pro/eNKI_RS_TRT/FrontPage.html) (Nooner et al., 2012). This dataset includes multi-modal imaging data of 24 participants (age: $34.4 \pm 12.9,6$ females), which have been recently used in the test-retest reliability studies of functional homogeneity (Zuo et al., 2013), voxel-wise functional connectivity (Liao et al., 2013), and directed brain network analysis (Wu et al., 2013). In the current study, we discarded the data of 13 participants due to the potential effects of confounding health issues (current/historical psychiatric disorders, obvious brain atrophy or missing diagnostic information) or because of excessive head motion (see data preprocessing) (Table S1). Finally, the data of the remaining 11 healthy participants (age: $30.2 \pm 9.6,4$ females) were used for subsequent network analyses (Table S1).

\section{Data Acquisition}

All of the participants underwent both R-fMRI and DTI scans twice, approximately 1 week apart, on a Siemens Trio 3.0 Tesla scanner, leading to repeated data in two sessions (i.e., Session 1 and Session 2). In each session, the R-fMRI scans were performed using three echo planar imaging protocols, each with different sampling rates ( $\mathrm{TR}=645,1400$, and $2500 \mathrm{~ms}$ ), and participants were instructed to keep their eyes fixed on the cross on the screen. Here, we employed the multiband R-fMRI data with the sub-second sampling rate $(\mathrm{TR}=645 \mathrm{~ms}$ ), which provided finer temporal information regarding fluctuations in the BOLD 
signals. The detailed scanning parameters were as follows: time repetition $[\mathrm{TR}]=645 \mathrm{~ms}$; time echo $[\mathrm{TE}]=30 \mathrm{~ms}$; flip angle $=60^{\circ} ; 40$ slices, multiband acceleration factor $[\mathrm{MAF}]=4$; field of view $[\mathrm{FOV}]=222 \times 222 \mathrm{~mm}^{2}$; voxel size $=3 \times 3 \times 3 \mathrm{~mm}^{3}$; total acquisition time $=9: 46 \mathrm{~min}$ (i.e., 900 volumes). Because the last (900th) functional volume was missing in the R-fMRI data of four participants, we used the 899 volumes available for all participants. The parameters for the multiband DTI images were as follows: $\mathrm{TR} / \mathrm{TE}=2400 / 85 \mathrm{~ms}$; flip angle $=90^{\circ}$; 64 slices, $\mathrm{MAF}=4 ; \mathrm{FOV}=212 \times 180 \mathrm{~mm}^{2}$; voxel size $=2 \times 2 \times 2 \mathrm{~mm}^{3}$; $b$-value $=1500 \mathrm{~s} / \mathrm{mm}^{2}, 128$ gradient directions with $9 \mathrm{~b}=0$ images; total acquisition time $=5: 58 \mathrm{~min}$. Notably, DTI data of one participant was missing in the first session (Table S1). Additionally, a high-resolution T1-weighted image was also obtained for each participant with a magnetization prepared rapid gradient echo (MPRAGE) sequence: TR/TE $=2500 / 3.5 \mathrm{~ms}$; flip angle $=8^{\circ}$; inversion time $=1200 \mathrm{~ms} ; 192$ slices; Matrix $=$ $256 \times 256$; voxel size $=1 \times 1 \times 1 \mathrm{~mm}^{3}$. In the present study, the data from Session 1 were used for the main analyses, and the data from Session 2 were used for the validation analyses.

\section{Data Preprocessing and Analysis Multiband R-fMRI Data}

Data preprocessing was performed using Statistical Parametric Mapping (SPM8, http://www.fil.ion.ucl.ac.uk/spm) and Data Processing Assistant for Resting-State fMRI (DPARSF) (Yan and Zang, 2010). First, 16 volumes in the first $10 \mathrm{~s}$ were discarded for signal equilibrium and to allow the participants' adaption to the scanning environment. The remaining data were corrected for head motion and participants with large head motion $(2 \mathrm{~mm}$ or $>$ $2^{\circ}$ ) were excluded (Table S1). Subsequently, the motion-corrected images were spatially normalized to Montreal Neurological Institute (MNI) space using an optimum, 12-parameter affine transformation and nonlinear deformations (Ashburner and Friston, 1999) and then resampled to 3-mm isotropic voxels. Linear trends were further removed from the normalized images, and then the images were temporally band-pass filtered (0.01$0.1 \mathrm{~Hz}$ ). Finally, several confounding factors were regressed out as covariates using multiple linear regression, including 24 headmotion parameters (Friston et al., 1996) and cerebrospinal fluid (CSF), white matter (WM) and global brain (Fox et al., 2005) signals. The residual time series were used for further network analysis.

\section{Multiband DTI Data}

The preprocessing of the DTI data consisted of eddy current and motion artifact correction, estimation of the diffusion tensor, calculation of fractional anisotropy (FA) and diffusion tensor tractography. The first three steps were performed with the FDT toolbox in FSL (http://www.fmrib.ox.ac.uk/fsl) (Smith et al., 2004) as follows. First, an affine transformation was applied to align each diffusion-weighted image to the $b=0$ image to correct for eddy current distortions and motion artifacts. Second, the diffusion tensor was calculated by solving the Stejskal and Tanner equation (Basser et al., 1994), and the reconstructed tensor matrix was diagonalized to obtain three eigenvalues $(\lambda 1$, $\lambda 2$, and $\lambda 3$ ) and corresponding eigenvectors. Finally, the FA value was calculated voxel by voxel using the three eigenvalues of the tensor matrix (Basser and Pierpaoli, 1996). Subsequently, the structural T1-weighted image was segmented into gray matter (GM), WM and CSF in the CIVET pipeline (http://mcin-cnim. $\mathrm{ca} /$ neuroimagingtechnologies/civet/) and registered to the $b=0$ image to obtain the white matter mask in DTI native space. Diffusion tensor tractography was then implemented in Diffusion Toolkit (http://trackvis.org/) using the "fiber assignment by continuous tracking (FACT)" method (Mori et al., 1999) by seeding each voxel of the white matter mask. Specifically, given a voxel, eight seeds were placed evenly within the volume of the voxel (van den Heuvel and Sporns, 2011). Then each fiber was reconstructed by tracking from each seed following the main diffusion direction of the current voxel into the next, thus reconstruction was terminated if the fiber turned with an angle $>45^{\circ}$ or went out of the white matter mask (Mori et al., 1999). After tracking from all white matter voxels of the brain, a large member of fiber bundles was reconstructed and formed the whole-brain WM fibers for each participant.

\section{Regional Parcellations}

To derive inter-regional functional and structural connectivity, we parcellated the cerebral cortex into different regions of interest (ROIs). Given that the topological organization of the brain networks could be affected by different spatial parcellations (Wang et al., 2009; Fornito et al., 2010; Zalesky et al., 2010), in the present study we investigated the functional and structural connectomics using two different regional parcellation schemes representing low and high spatial resolution, respectively. In the first parcellation scheme, 90 ROIs were obtained based on the automated anatomical labeling atlas (AAL-90; for details of the ROIs, see Table S2) (Tzourio-Mazoyer et al., 2002); in the second parcellation scheme, 1024 ROIs with uniform sizes were obtained based on a random parcellation (H-1024) (Zalesky et al., 2010). For each parcellation scheme, we investigated the dynamic functional connectivity (D-FC) and structural connectivity (SC) among regions. Notably, the analysis of the H-1024 brain networks was identical to that of the AAL-90 brain networks.

\section{Dynamic Functional Connectivity Analysis Extraction of D-FC}

For each participant, we employed a commonly used sliding window approach to estimate the D-FC of every pair of ROIs (Kiviniemi et al., 2011; Jones et al., 2012; Tagliazucchi et al., 2012b; Hutchison et al., 2013b; Allen et al., 2014; Zalesky et al., 2014). Briefly, the time course of each ROI was first obtained by averaging the residual time courses of all voxels within the ROI. Then, a sliding rectangular window with a fixed length was selected, and each ROI's time course within this window was used to estimate the D-FC of interest. Within a given sliding window $t$, a symmetric $N \times N$ D-FC matrix, $R_{t}=\left[r_{t}(i, j)\right]$, was generated, where $N$ denotes the number of ROIs considered ( $N=90$ for the AAL-90 network and $N=1024$ for the $\mathrm{H}$ 1024 network), and $r_{t}(i, j)$ represents the Pearson's correlation coefficient between the time courses of two ROIs, $i$ and $j$. Here, we utilized a sliding window with a length of 155 TRs (i.e., 100 s), which allows us to estimate D-FC over the low-frequency band of 
interest $(0.01-0.1 \mathrm{~Hz})$ with an adequate number of time points (at least one period), and simultaneously to capture the time-varying information of the D-FC. This window was shifted in time with a step size of one TR (i.e., $645 \mathrm{~ms}$ ), resulting in $729 \mathrm{D}$-FC matrices $\left(R_{t}, t=1,2, \ldots, 729\right)$ for each participant (Figure 1; Video S1). We also assessed the effects of different sliding window lengths on the main results (see "Validation analysis").

\section{Temporal Characteristics of D-FC}

We studied the time-varying features of the D-FC matrices as follows: (i) To estimate the temporal fluctuations of the whole-brain D-FC patterns, for each participant, we constructed the $N \times N$ static functional connectivity (S-FC) matrix as a reference, which was represented by Pearson's correlation coefficients over the whole-scan time courses. The time-varying spatial similarity between the S-FC and D-FC matrices, $R_{(\mathrm{S}-\mathrm{FC}, \mathrm{D}-\mathrm{FC})}$, was calculated via Pearson's correlation analysis within each sliding window. Notably, prior to the correlation analysis, Fisher's r-to-z transformation (Zar, 1996) was performed on all entries in both matrices to improve the normality of the correlation distribution. (ii) To quantitatively ascertain the temporal features of the D-FC between every pair of ROIs ( $i$ and $j$ ), two measures, temporal mean (tMean) and temporal variability (tVar), were estimated as follows:

$$
\begin{array}{r}
\operatorname{tMean}_{\mathrm{D}-\mathrm{FC}(\mathrm{i}, \mathrm{j})}=\frac{1}{T} \sum_{t=1}^{T} r_{t}(i, j), \\
t \operatorname{Var}_{\mathrm{D}-\mathrm{FC}(\mathrm{i}, \mathrm{j})}=\sqrt{\frac{1}{T-1} \sum_{t=1}^{T}\left(r_{t}(i, j)-\operatorname{tMean}_{\mathrm{D}-\mathrm{FC}(\mathrm{i}, \mathrm{j})}\right)^{2}},
\end{array}
$$

where, $r_{t}(i, j)$ represents the D-FC strength between ROIs $i$ and $j$ within a given window $t$, and $T$ denotes the total number of sliding windows (here, $T=729$ ) for each participant. Therefore, for each participant an $N \times N$ D-FC strength matrix and an $N \times N$ D-FC variability matrix were generated. (iii) To further explore the spatial dependence of D-FC strength and variability, we generated the average $\mathrm{D}$-FC strength and variability matrices across individuals and then classified all of the D-FCs into

A

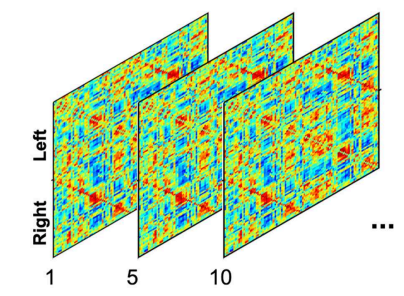

Time-varying functional connectivity matrices (ID: 9)

B Spatial similarity between S-FC and D-FC (ID:9)

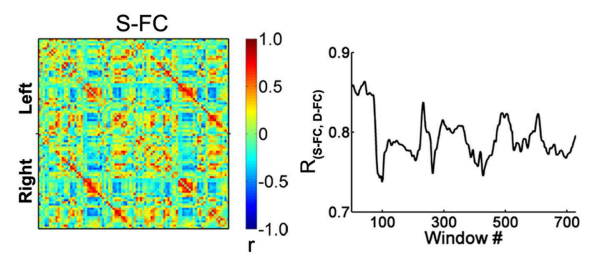

C Spatial similarity between S-FC and D-FC (All)
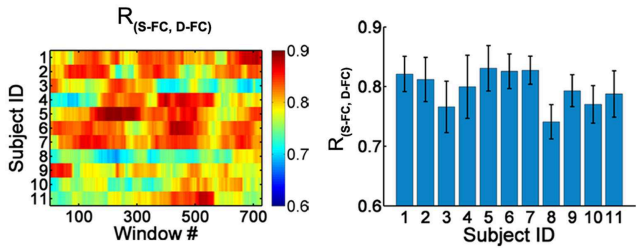

D D-FC strength and its spatial dependence

\section{E D-FC variability and its spatial depencence}
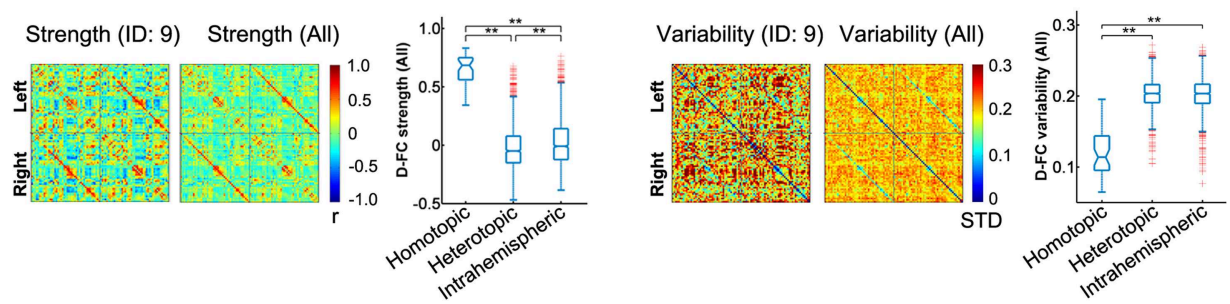

FIGURE 1 | Spatial patterns of D-FC matrices over time. (A) Transient D-FC matrices at different sliding windows for one representative participant (ID: 9). Regions of interest (ROIs) were indexed in an order of left (ROls: 1-45) to right (ROls: 46-90) hemisphere (for details of the ROls, see Table S2). (B) Static functional connectivity (S-FC) matrix of the same participant (ID: 9) (left) and its spatial similarity with the D-FC matrices over time (right). $R_{(S-F C, D-F C)}$ denotes the spatial similarity between the S-FC and the D-FC matrices obtained using Pearson's correlation analysis following Fisher's r-to-z transformation. (C) Time-varying spatial similarity between the S-FC and the D-FC matrices for all participants (left) and associated temporal mean and temporal variability across windows (right). Error bars represent the standard deviation across windows. (D) D-FC strength and its spatial dependence for one participant (ID: 9) and for all participants. (E) D-FC variability and its spatial dependence for one participant (ID: 9) and for all participants. In (D, E), the D-FC strength/variability matrix for all participants was generated by averaging the individual strength/variability matrices across participants. The statistical difference between any two categories of D-FC was tested using permutation tests $\left({ }^{\star *}\right.$ Bonferroni corrected $p<0.001,10,000$ permutations). S-FC, static functional connectivity; D-FC, dynamic functional connectivity. 
three broad categories based on the inter-regional spatial relationships (Stark et al., 2008): homotopic edges, representing the connections between homologous brain areas in two hemispheres; heterotopic edges, representing the connections between non-homologous brain areas in two hemispheres; and intrahemispheric edges, representing the connections between brain areas within the same hemisphere. Next, differences in DFC strength or variability between any two edge categories were tested using permutation tests, during which the D-FC group assignments were randomly shuffled 10,000 times to obtain the null distribution. The Bonferroni approach was applied to correct for multiple testing across all of the category pairs examined (three in total).

\section{Topological Analyses of Dynamic Functional Brain Networks \\ Construction of Dynamic Functional Networks}

To assess the time-varying topological properties of whole-brain functional networks, for each participant, we first generated a set of binary D-FC matrices (i.e., binary functional networks) over time through a thresholding approach and then performed graph-based network analysis. Specifically, for a given sliding window $t$, any correlation $r_{t}(i, j)$ greater than a given threshold $\tau$ was retained as an edge connecting ROIs $i$ and $j$; no edge existed otherwise. The correlation threshold $\tau$ was determined via a Bonferroni correction with $p_{\text {corr }}<0.01$, which preserved the significantly existing correlations, producing dynamic functional networks with different edge numbers. Moreover, the edges with negative correlations were ignored during the network topology analysis, as their physiological meaning is not clear yet (Fox et al., 2009; Murphy et al., 2009; Weissenbacher et al., 2009; Adachi et al., 2012). We also assessed the effect of different thresholding strategies on the main results (see "Validation analysis").

\section{Network Topology Analysis}

All network analyses were performed using the in-house Gretna package (http://www.nitrc.org/projects/gretna). For the D-FC network within each sliding window, we calculated both global and nodal network metrics (for review, see Rubinov and Sporns, 2010), to characterize their overall architecture and nodal centrality, respectively. (i) The global network metrics included connectivity density or sparsity $(S)$, small-world attributes (clustering coefficient, $C_{p}$, and characteristic path length, $L_{p}$ ), assortativity $(\alpha)$, and their normalized versions using random networks $\left(\gamma, \lambda\right.$ and $\left.\alpha_{z-\text { score }}\right)$. Briefly, the density or sparsity $(S)$ of a network characterizes the number of existing edges, indicating the total wiring costs of the network. The clustering coefficient of a node reflects the fraction of triangles around this node, indicating the extent of local information segregation (Watts and Strogatz, 1998). Hence the clustering coefficient $\left(C_{p}\right)$ of a network characterizes the prevalence of local clustering in the network, reflecting the overall capability for information segregation. The characteristic path length $\left(L_{p}\right)$ describes the average length of the shortest paths linking any two nodes in the network, measuring the capability for information integration. Typically, a small-world network architecture supports the balance between information segregation and integration with a low wiring cost, and is characterized by high local clustering $(\gamma=$ $\left.C_{p} /<C_{p}^{\text {rand }}>>>1\right)$ and short path length $\left(\lambda=L_{p} /<L_{p}^{\text {rand }}>\sim\right.$ 1 ), leading to a small-world index $\sigma=\gamma / \lambda>1$ (Humphries and Gurney, 2008). Specifically, $\left\langle C_{p}^{\text {rand }}>\right.$ and $<L_{p}^{\text {rand }}>$ were the average of $C_{p}$ and $L_{p}$, respectively, estimated from 100 matched random networks conserving the same number of nodes, edges and the same degree distribution as the real brain networks (Maslov and Sneppen, 2002). Assortativity $(\alpha)$ describes the similarity of degree values for any two connected nodes (Newman, 2002). Positive values of $\alpha$ indicate nodes possessing large degrees prefer to connect with each other to form a relatively resilient core, and vice versa. Assortativity is considered significant if the normalized value $\alpha_{z-\text { score }}=$ $\left(\alpha^{\text {real }}-<\alpha^{\text {rand }}>\right) / s t d\left(\alpha^{\text {rand }}\right)>1.64$ (i.e., one-tailed $t$-test with $p<0.05)$, where $<\alpha^{\text {rand }}>$ and $\operatorname{std}\left(\alpha^{\text {rand }}\right)$ denote the mean and the standard deviation of the $\alpha$ values, respectively, estimated from 100 matched random networks. (ii) For regional topological properties, we employed nodal degree centrality due to its higher test-retest reliability in the functional networks than other nodal metrics (Wang et al., 2011). The degree $k_{i}$ of node $i$ is defined as the number of edges directly connected to this node, indicating its role in the information communication in the network.

\section{Temporal Characteristics of Network Topology}

The temporal characteristics of each network metric were estimated across windows within each participant, which were further averaged across individuals for group-level analysis. Briefly, for each global metric, including $S, C_{p}, L_{p}, \gamma, \lambda, \sigma$, and $\alpha$, we estimated a normalized histogram across windows. For nodal degree, we identified the hub regions for each sliding window, the degree values of which exceeded the mean value across the brain. Then, we counted the occurrence probability (i.e., ratio of sliding windows) as functional hubs for each region, and identified functionally persistent hub regions that possessed occurrence probability $>50 \%$ over time. Finally, we estimated the temporal mean (tMean) and temporal variability $(t$ Var $)$ of each global or nodal metric over time as follows:

$$
\begin{array}{r}
\operatorname{tMean}_{N E T}=\frac{1}{T} \sum_{t=1}^{T} N E T_{t}, \\
t \operatorname{Var}_{N E T}=\sqrt{\frac{1}{T-1} \sum_{t=1}^{T}\left(N E T_{t}-t \text { Mean }_{N E T}\right)^{2},}
\end{array}
$$

where, NET represents the global or nodal metric of interest, $N E T_{t}$ denotes its value for a given sliding window $t$, and $T$ denotes the total number of sliding windows (here, $T=729$ ) for each participant.

\section{Relationship between Structural and Dynamic Brain Networks}

Recent studies have suggested that the intrinsic or spontaneous functional organization of the brain is shaped by the underlying structural architecture (Hagmann et al., 2008; Honey et al., 2009; See Reviews Deco et al., 2011, 2013; Park and Friston, 2013; Wang et al., 2015). Here, we reconstructed human white 
matter structural networks using DTI data from the same participants, and then investigated the relationship between dynamic functional networks and structural networks at different levels (including connectional, global and nodal properties).

\section{Construction of Structural Networks}

The network construction approaches were the same as our previous procedures (Gong et al., 2009; Shu et al., 2011). Briefly, the nodes were first obtained using SPM8 (http://www.fil.ion. ucl.ac.uk/spm) based on the parcellation schemes mentioned above (AAL-90 or H-1024). Specifically, the registered T1 image was nonlinearly transformed into the ICBM152 T1 template in the MNI space, and the corresponding transformation matrix was inversed to warp the parcellation scheme (AAL-90 or H1024) from the MNI space to the DTI native space using a nearest-neighbor interpolation method to preserve discrete labeling values. Second, any two ROIs in DTI native space were considered structurally connected if at least one fiber streamline existed with two end-points locating in these two regions, respectively (Shu et al., 2011; Zalesky et al., 2011). Hence, for each participant, an undirected weighted SC matrix was constructed, indicating the presence or absence of SC between region pairs, as well as the SC strength. Specifically, for each SC its strength was denoted by the normalized number of streamlines, which was calculated by dividing the number of interconnecting streamlines by the mean volume of the two connected regions. Additionally, to balance the inter-individual variance in structural connections, we identified significantly consistent connections across participants by performing a nonparametric one-tailed sign test on the normalized streamline number (Gong et al., 2009). For each pair of ROIs, the sign test was performed with the null hypothesis that no connection existed $(p<0.05)$. Non-zero connections were preserved and assigned the average SC strength across participants to generate the group-level SC network (i.e., backbone).

\section{Relationship between D-FC and SC Networks}

We explored the relationship between D-FC and SC networks at the following three levels. (i) Connection level. The relationship between D-FC and SC was performed at both individual and group levels. Specifically, for each participant, we examined whether there was a significant difference in D-FC strength or variability between region pairs with direct SC and those without direct SC. Here, a non-parametric permutation test was employed, where D-FC group assignments were randomly shuffled 10,000 times between the two categories of SC present and SC absent. Then, for the region pairs with direct SC, the relationship between SC strength and D-FC strength or variability was estimated using Pearson's correlation analysis. Prior to correlation analysis, a Gaussian resampling method was separately applied to all of the measures to improve the normality of their distributions. Similar analysis was also performed at the group level, based on the SC backbone network. (ii) Globaltopology level. For each participant, we first estimated the global network metrics, including $C_{p}, L_{p}, \gamma, \lambda, \sigma$, and $\alpha$, for the binary structural networks. Then, for each global metric, we used an across-subject Pearson's correlation analysis to examine the relationship between the SC networks and the temporal features of the D-FC networks, including temporal mean and temporal variability. (iii) Nodal-topology level. For each participant, we calculated nodal degree centrality in the binary structural network and identified the structural hub regions whose degree values exceeded the mean value across the brain. Then, we counted the occurrence probabilities of structural hubs across individuals, and identified structurally consistent hub regions with occurrence probabilities $>50 \%$. Furthermore, for all participants, we explored the potential constraints of both structural hub probability and structural degree centrality on the temporal variability of functional degree centrality via acrossnode correlation analysis. Because the values of structural hub probability were discrete, Spearman's correlation analysis was performed in these correlation analyses. Notably, during steps (ii) and (iii), network metrics were estimated for binary structural networks ignoring the SC strength, to ensure the comparability of network topology between structural and dynamic functional networks.

\section{Validation Analysis}

We evaluated whether the main findings were affected by scanning session or by different analysis strategies (e.g., the window length, the correlation thresholding strategy for network construction, and the network type). Given that the main results were compatible for low-resolution (AAL-90) and highresolution (H-1024) brain networks (see Results), the validation analyses were performed on the low-resolution AAL-90 networks to reduce the computational burden. The relevant procedures are described as follows: (i) Scanning session. To validate our main findings, we performed the same network analysis on the multiband R-fMRI and DTI dataset in Session 2 of the same participants, which was scanned approximately 1 week after Session 1. (ii) Window length. In the current study, a commonly used sliding window approach was employed to capture the dynamics of D-FC (Kiviniemi et al., 2011; Jones et al., 2012; Tagliazucchi et al., 2012b; Hutchison et al., 2013b; Allen et al., 2014; Zalesky et al., 2014). However, so far, the choice of window length remains controversial (Hutchison et al., 2013a); various window lengths have been used in the previous studies (e.g., $30 \sim 240$ s in Hutchison et al., 2013b). In the main analyses, we employed a sliding window with a length of $100 \mathrm{~s}$, to capture the primary low-frequency BOLD signal fluctuations. Moreover, two additional window lengths (50-150 s) were considered to validate the main results. (iii) Correlation thresholding strategy. During functional network construction, various strategies can be used to threshold the functional correlation matrix to derive a connectivity matrix representing the functional network (Wang et al., 2010). Here, we employed a fixed correlation threshold for all of the windows, which was determined by a significance criterion-Bonferroni correction with $p_{\text {corr }}<0.01$. To assess the effect of different correction thresholds, we conducted analyses at two additional thresholds of significance $p_{\text {corr }}<0.05$ and 0.001 . Moreover, we also generated the D-FC brain networks using different connectivity densities or sparsities (10, 15, and 20\%), to ensure the same number of connections in the D-FC networks across all of the windows. (iv) Network type. In the main 
analyses, the network analyses were performed on binary brain networks, ignoring the differences in edge weights or connection strength. To assess whether our main results depended on the edge weights, we performed the same graph-based analyses on weighted D-FC networks and structural networks, where the weighted D-FC networks were obtained using the Bonferroni correction approach $\left(p_{\text {corr }}<0.01\right)$, but retaining the correlation values for the significant connections.

\section{Results}

In the current study, we constructed dynamic functional networks and structural networks using both low-resolution (AAL-90) and high-resolution (H-1024) parcellations, and further investigated the dynamic characteristics of the functional networks and their associations with structural connectivity features. Given that most of the findings obtained from the AAL-90 and H-1024 brain networks were compatible, we mainly reported the results from the AAL-90 brain network analyses.

\section{Dynamic Functional Connectivity among Regions} Using the sliding window approach, we generated individual inter-regional D-FC matrices, fluctuating at a sub-second time scale, for the whole brain. Figure 1A illustrates the transient D-FC matrices of several windows (i.e., time) for one representative participant (ID: 9; see Video S1 for all of the D-FC matrices across time). Notably, although the $\mathrm{D}$-FC matrices varied across windows, they maintained high spatial similarity $\left[R_{(\mathrm{S}-\mathrm{FC}, \mathrm{D}-\mathrm{FC})}>0.7\right]$ with the S-FC matrix (Figure 1B). Similar features were also observed for other participants (Figure 1C), with the mean $R_{(\mathrm{S}-\mathrm{FC}, \mathrm{D}-\mathrm{FC})}$ across windows exceeding 0.7 , suggesting the existence of intrinsic rules (e.g., potential structural constraints) underlying the dynamic functional coordination. To further ascertain the temporally fluctuating characteristics of these D-FC matrices, for each participant, we computed the connectivity strength and temporal variability of each D-FC and obtained the D-FC strength and variability matrices, respectively (Figures 1D,E). At the group level, we found that homotopic D-FC showed significantly greater connectivity strength and smaller temporal variability than either heterotopic or intrahemispheric D-FC (both Bonferroni-corrected $p s<0.001,10,000$ permutations).

\section{Dynamic Global Properties of Functional Brain Networks}

To assess the time-varying topological properties of the functional networks, for each participant, we first generated a set of binary D-FC matrices over time using a thresholding approach (Bonferroni correction, $p_{\text {corr }}<0.01$ ), and then performed graphbased network analyses. We showed that the global topological properties $\left(S, C_{p}, L_{p}, \gamma, \lambda, \sigma\right.$, and $\left.\alpha\right)$ of the D-FC brain networks fluctuated over time (Figure 2A and Table S3) and obtained corresponding normalized histograms across windows (Figure 2B). First, for all participants, network connectivity density or sparsity $(S)$ fluctuated within a similar range $(0.10-$ 0.24 ), with the distribution centered at approximately 0.17 . Out of the 8019 (i.e., 11 participants $\times 729$ windows) DFC networks obtained, $97.8 \%$ were fully connected, and the remaining networks had at least 88 nodes connected (Table S4). Second, all of the D-FC networks exhibited a clustering coefficient $\left(C_{p}\right)$ above 0.4 and a characteristic path length $\left(L_{p}\right)$ below 2.4. Compared to matched random networks, these D-FC networks were more locally clustered (i.e., $\gamma=C_{p}^{b r a i n} / C_{p}^{\text {rand }}>>$ 1) and had an almost identical path length (i.e., $\lambda=L_{p}^{\text {brain }} / L_{p}^{\text {rand }}$ $\sim 1$ ) for all windows, indicating dynamic, but persistent smallworld architecture over time (all $\sigma \mathrm{s}>1.6$ ). Third, the temporal fluctuations of assortativity $(\alpha)$ had a distribution centered at approximately 0.35 (all $\alpha_{z-\text { score }}>1.64$ ), suggesting that nodes that possessed a similar number of edges tended to connect with each other.
A

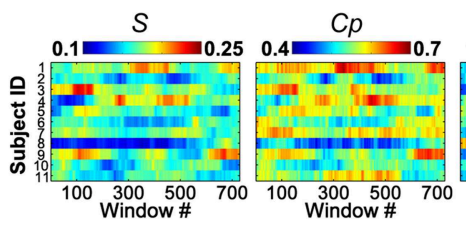

B
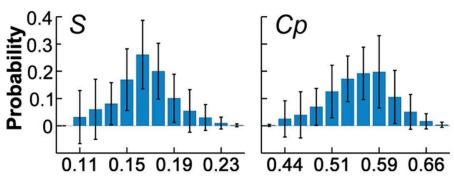

Time-varying global network properties (All)

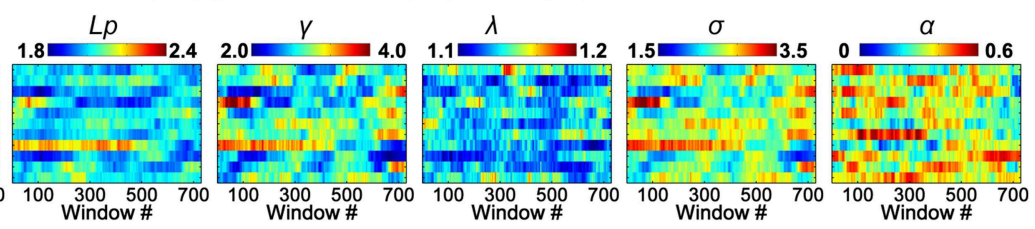

Normalized histograms across windows (All)
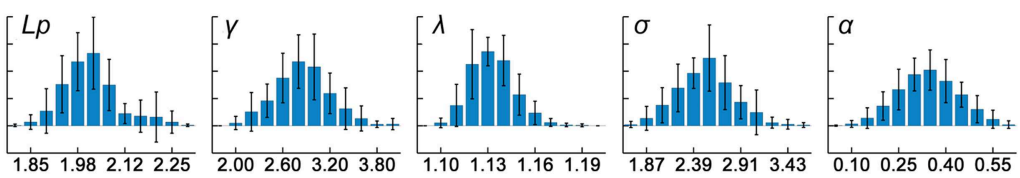

FIGURE 2 | Dynamic global topological properties of D-FC brain networks. (A) Time-varying global network properties for all participants, including sparsity $(S)$, clustering coefficient $\left(C_{p}\right)$, characteristic path length $\left(L_{p}\right)$, normalized clustering coefficient $(\gamma)$, normalized characteristic path length $(\lambda)$, small-worldness $(\sigma)$, and assortativity $(\alpha)$. (B) Normalized histograms of global properties across windows for all participants. For each metric, a normalized histogram across windows was estimated for each participant; these histograms were then averaged across participants to generate the mean histogram. Error bars represent the standard deviation of the normalized histograms across participants. D-FC, dynamic functional connectivity. 


\section{Dynamic Hubs in Functional Brain Networks}

Using nodal degree centrality, we assessed the nodal roles in information communication of the D-FC networks. For each participant, the degree centrality of brain regions fluctuated over time, reflecting dynamic reconfiguration of spatial patterns (Figure 3A and Video S2 for one representative participant, ID: 9). Further, we identified transient functional hubs (above the mean) for each sliding window and estimated their occurrence probabilities across windows. For the participant with ID 9, we found that several regions appeared as persistent hubs across time with large probabilities $(>0.5)$, which were mainly located at the insula, sensorimotor cortex, superior occipital gyrus, superior temporal gyrus, medial prefrontal cortex, and anterior and middle cingulate cortex (Figure 3A). At the group level, these regions were also identified as persistent functional hubs over time (Figure 3B, Table 1). Finally, the across-node correlation analysis revealed a significant positive association between the occurrence probability as hubs and the temporal variability of nodal degree centrality in the D-FC networks $(r=0.41, p<$ 0.0001 ; Figure 3B), indicating that functionally persistent hubs (with denser connectivity across time) tended to exhibit larger temporal variability.

\section{Relationship between Dynamic Functional Networks and Structural Connectivity Networks Connectivity Relationship between D-FC and SC}

Using DTI tractography, we reconstructed the white matter fibers in the whole brain for each participant, and then generated individual SC networks. Figure 4A displays the whole-brain white matter fibers and corresponding structural network for one representative participant (ID: 9). We found that regional pairs with direct SC exhibited significantly larger D-FC strength and lower D-FC temporal variability than those without direct SC (Figure 4A, both $p s<0.0001,10,000$ permutations). Moreover, for those region pairs with direct SC, D-FC strength was positively correlated with SC strength $(r=0.24, p<$ $0.0001)$, whereas D-FC variability was negatively correlated with SC strength $(r=-0.14, p<0.0001)$. Similar results were also observed for other participants (Table 2) and for the group-level analysis based on the SC backbone network (Figure 4B).

\section{Topological Relationship between D-FC and SC Networks}

We calculated both global and nodal topological properties for individual binary SC networks and examined their associations with the temporal features of D-FC networks (e.g., temporal mean and variability of the topological properties across the sliding windows). First, we found that all of the individual SC networks exhibited small-world architecture (mean \pm std: $\sigma=1.98 \pm 0.13)$, characterized by dense local clustering $(\gamma>1)$ and short path length $(\lambda \sim 1)$ (Table 3). Meanwhile, assortative organization was also observed for each participant, accompanied by $\alpha_{z \text {-score }}>1.64$ (one-tailed $p<0.05$ ). Notably, for each global metric $\left(C_{p}, L_{p}, \gamma, \lambda, \sigma\right.$, and $\left.\alpha\right)$, its temporal mean and variability in D-FC networks did not exhibit significant across-subject correlation ( $p$ s $>0.05$ ) with the SC networks.
A

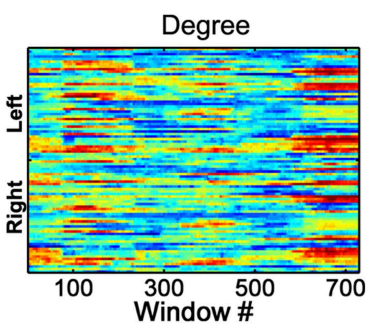

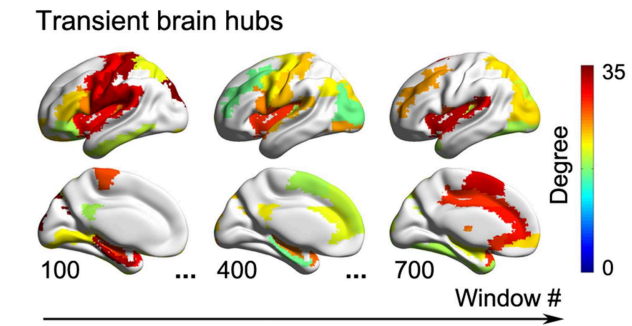

Time-varying degree centrality and dynamic functional brain hubs (ID: 9)

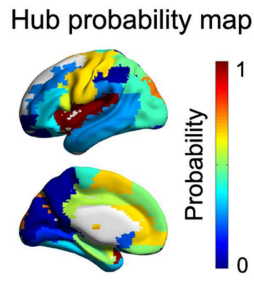

B
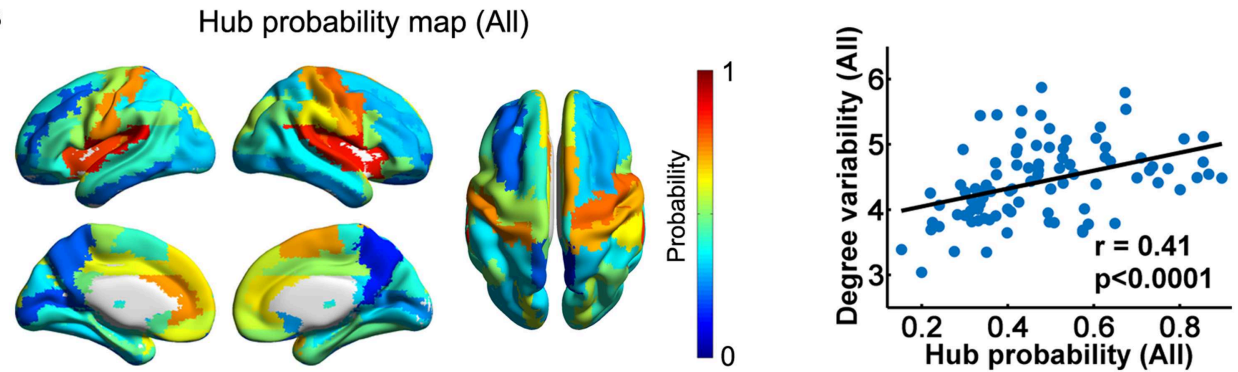

FIGURE 3 | Dynamic functional hubs in the D-FC brain networks. (A) Time-varying degree centrality and dynamic functional brain hubs for one representative participant (ID: 9). Transient brain hubs at several windows (e.g., \# =100, 400, and 700) are displayed. Hub probability map was generated by counting the occurrence probability as hubs across sliding windows for each region. (B) Hub probability map for all participants (left) and scatter plot showing the across-node relationship between hub probability and temporal variability of functional degree centrality (right). The probability map for all participants was generated by averaging individual hub probability maps across participants. The surface-based visualizations were made using BrainNet Viewer (http://www.nitrc.org/projects/bnv/, Xia et al. 2013). D-FC, dynamic functional connectivity. 
TABLE 1 | Functionally persistent hubs in dynamic brain networks (AAL-90).

\begin{tabular}{|c|c|c|}
\hline ROI name & $\begin{array}{c}\text { Hub } \\
\text { probability }\end{array}$ & $\begin{array}{c}\text { Degree variability } \\
\text { (z-score) }\end{array}$ \\
\hline L Rolandic operculum & 0.90 & 0.09 \\
\hline L Superior temporal gyrus & 0.87 & 0.19 \\
\hline R Insula & 0.85 & 1.18 \\
\hline R Rolandic operculum & 0.85 & 0.51 \\
\hline R Superior temporal gyrus & 0.84 & 0.10 \\
\hline L Insula & 0.81 & 1.12 \\
\hline R Heschl gyrus & 0.80 & -0.22 \\
\hline R Precental gyrus & 0.77 & 0.34 \\
\hline L Heschl gyrus & 0.75 & -0.03 \\
\hline L Postcentral gyrus & 0.74 & 0.39 \\
\hline L Anterior cingulate and paracingulate gyri & 0.73 & 0.29 \\
\hline R Supplementary motor area & 0.71 & 0.62 \\
\hline R Postcentral gyrus & 0.70 & 0.10 \\
\hline R Lenticular nucleus, putamen & 0.67 & 1.90 \\
\hline R Temporal pole: superior temporal gyrus & 0.67 & 2.34 \\
\hline L Superior frontal gyrus, medial & 0.65 & -1.10 \\
\hline R Anterior cingulate and paracingulate gyri & 0.64 & 0.53 \\
\hline R Supramarginal gyrus & 0.63 & 0.90 \\
\hline L Lenticular nucleus, putamen & 0.63 & 0.64 \\
\hline L Temporal pole: superior temporal gyrus & 0.62 & 1.42 \\
\hline L Gyrus rectus & 0.60 & -0.07 \\
\hline L Median cingulate and paracingulate gyri & 0.60 & 1.14 \\
\hline R Superior frontal gyrus, medial & 0.59 & -1.12 \\
\hline L Superior occipital gyrus & 0.58 & -0.72 \\
\hline R Superior frontal gyrus, medial orbital & 0.57 & -1.28 \\
\hline L Superior frontal gyrus, medial orbital & 0.57 & -1.33 \\
\hline R Superior occipital gyrus & 0.55 & 0.19 \\
\hline L Precental gyrus & 0.54 & 0.44 \\
\hline L Paracentral lobule & 0.54 & 1.08 \\
\hline R Median cingulate and paracingulate gyri & 0.53 & 0.87 \\
\hline R Inferior frontal gyrus, opercular part & 0.53 & -0.04 \\
\hline L Inferior frontal gyrus, opercular part & 0.53 & 0.62 \\
\hline R Gyrus rectus & 0.52 & 0.35 \\
\hline L Angular gyrus & 0.51 & -1.09 \\
\hline
\end{tabular}

Functionally persistent hub regions (i.e., probability $>0.5$ ) at the group level are listed here. $L$, left; $R$, right. Hub probability represents the occurrence probability as functional hubs across windows, and degree variability denotes temporal variability of functional degree centrality across windows, which was further converted to $z$-score value by subtracting its mean across the brain and then dividing by the associated standard deviation. Positive $z$-score values indicate large temporal variability above the mean value across the brain.

Second, for the SC networks of each participant, we identified the pivotal hub regions with larger degree centrality (above the mean). Figure 5A displays the probability map of structural hubs across individuals: structurally persistent hubs (probability $>0.5$ ) were mainly located in the insula, medial prefrontal cortex, sensorimotor cortex, precuneus, middle temporal gyrus, and visual cortex (Table 4). Notably, some of these regions also appeared as functionally persistent hubs (Figure 3B), including the insula, medial prefrontal cortex, sensorimotor cortex, and superior occipital gyrus. Further across-node correlation analysis revealed that both structural hub probability $(r=-0.29, p=$ $0.0051)$ and structural degree centrality $(r=-0.28, p=$ 0.0083 ) were negatively correlated with the temporal variability of functional degree centrality in the D-FC networks (Figure 5B).

\section{Dynamic, High-resolution Functional Brain Networks}

Using the high-resolution regional parcellation (H-1024), we examined the temporal characteristics of the D-FC networks and relevant structural basis on a finer spatial scale (Figure 6 and Table S5). The main findings in the high-resolution networks were largely compatible with those observed in low-resolution (AAL-90) networks, which are outlined below: (i) Spatial patterns of D-FC. We found that the spatial patterns of the inter-regional $\mathrm{D}-\mathrm{FC}$ in the high-resolution brain networks remained relatively stable over time (see Video S3 for one represent participant, ID: 9), with homotopic D-FC exhibiting the highest strength and lowest temporal variability (Figure 6A). (ii) Topological properties of D-FC networks. For all participants, network connectivity sparsity $(S)$ fluctuated within a similar range $(0.05-$ 0.15 ), with the distribution centered at approximately 0.10 . Out of the 8019 (i.e., 11 participants $\times 729$ windows) D-FC networks obtained, $98.5 \%$ were fully connected, and the remaining networks had 1023 nodes connected (Table S4). We found that individual high-resolution D-FC networks exhibited timevarying, but persistent small-world and assortative architecture (Figure 6B), and possessed functional brain hubs that persisted over time (probability $>0.5$ ) (Figure 6C, and Video S4 for one representative participant, ID: 9). Notably, the spatial distribution of persistent hubs in the high-resolution D-FC networks was largely consistent with the distribution in the low-resolution brain networks, except that additional functional hubs were observed in the high-resolution D-FC networks in the posterior cingulate cortex and precuneus. (iii) Relationship between the D-FC and SC networks. First, at the connection level, region pairs with direct SC showed significantly greater D-FC strength and smaller D-FC variability than those without SC (both $p$ s $<0.0001,10,000$ permutations; Figure 6D). Moreover, for structurally connected regions, SC strength exhibited significant correlations with both D-FC strength $(r=0.32)$ and variability ( $r=-0.29$; Figure 6D, Table 2). Second, at the global topological level (small-world and assortativity properties), significant across-subject correlation was not observed ( $p s>$ 0.05 ) between individual SC networks and associated temporal features of D-FC networks. Finally, at the regional level, several structural brain hubs across individuals also appeared as functionally persistent hubs over time, such as the insula, medial prefrontal gyrus, somatosensory cortex, superior occipital gyrus, and precuneus (Figure 6E). Moreover, structural hubs with larger SC degree centrality tended to have lower D-FC degree variability across time ( $r=-0.11$; Figure 6E).

\section{Validation Results}

We validated our results by using different scanning sessions and different analysis strategies (involving the window lengths, correlation thresholding strategies and network types). (i) 

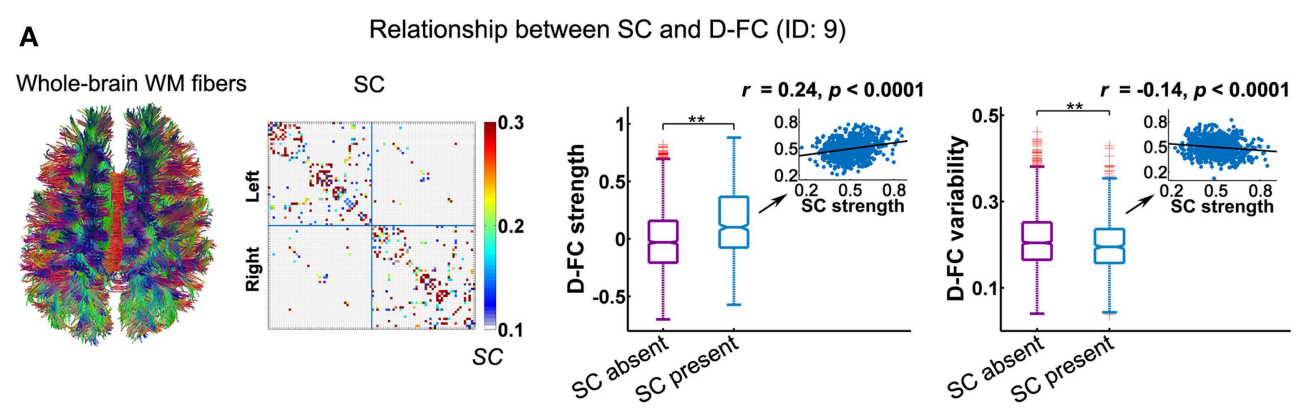

B

Relationship between SC and D-FC (All)
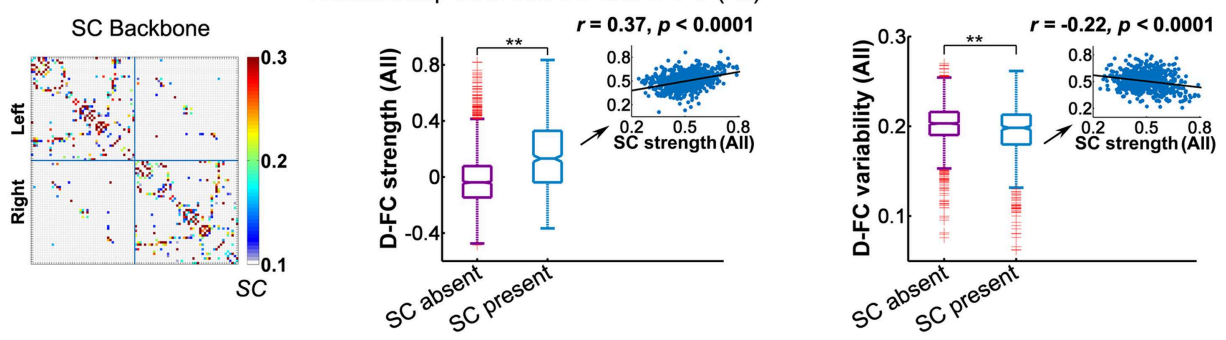

FIGURE 4 | Relationship between the D-FC and structural connectivity (SC) networks for one representative participant (ID: 9) (A) and for the whole group (B). (A) Schematic of whole-brain white matter (WM) fibers and SC matrix (left), and the differences in D-FC strength and variability between region pairs with direct SC (SC present) or not (SC absent) (right). The WM fibers were estimated from whole-brain tractography, and SC strength was represented by the normalized streamline number. (B) The SC backbone network across all participants (left) and differences in D-FC strength and variability between region pairs with or without direct SC (right). Group-level analyses were performed on the 10 participants that possessed both R-fMRI and DTI data, given that the DTI data of one participant (ID: 1) were not available. In (A,B), significant differences in D-FC strength/variability between two groups were tested using permutation tests ${ }^{* \star} p<0.0001,10,000$ permutations). Scatter plots (insets) show the correlation between D-FC strength/variability and SC strength, confined to the regional pairs with direct SC. Prior to the correlation analysis, all of these measures were resampled into a Gaussian distribution, with mean $\pm s t d=0.5 \pm 0.1$. D-FC, dynamic functional connectivity.

TABLE 2 | Relationship between dynamic functional connectivity and structural connectivity.

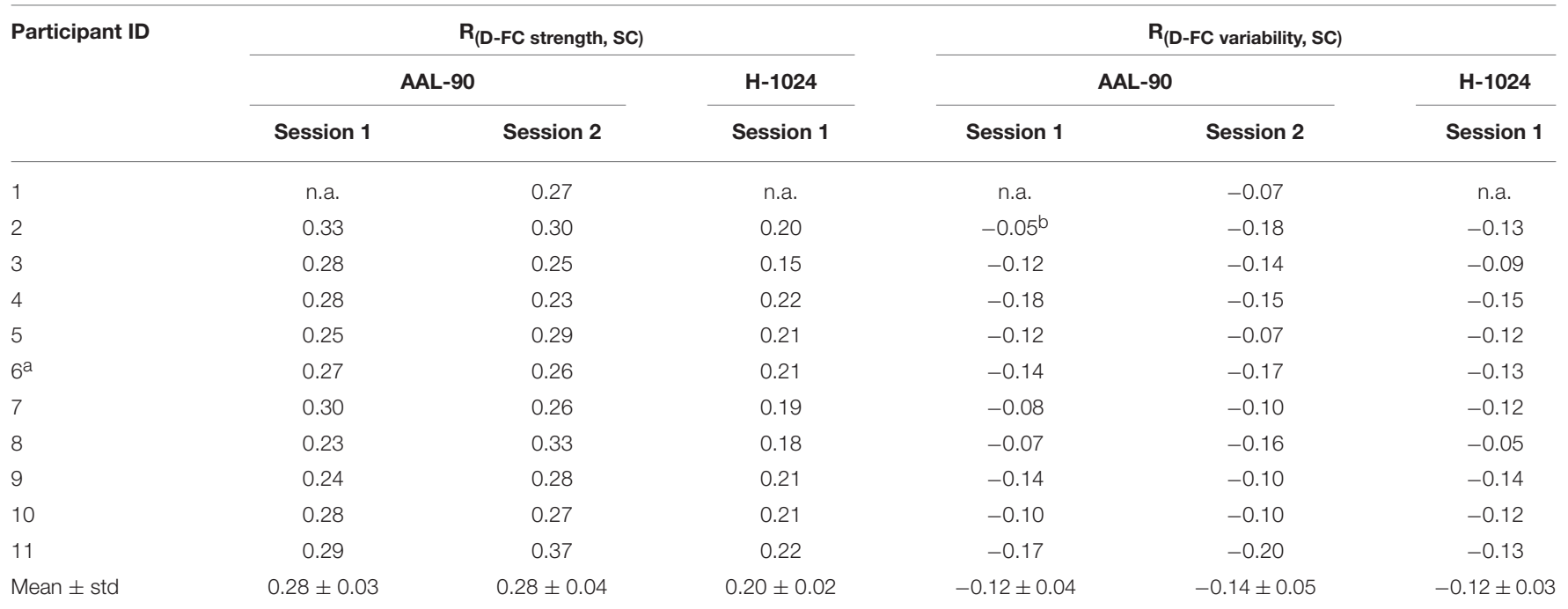

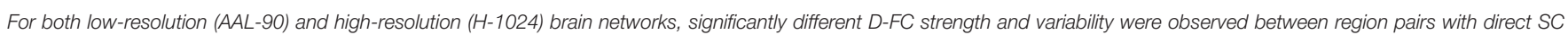

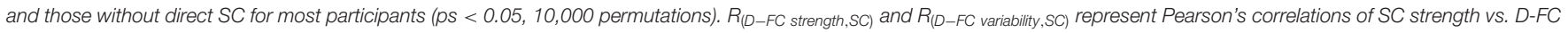
strength and D-FC variability, respectively, confined to region pairs with direct SC. Significant correlation $(p<0.05)$ was observed for almost all participants.

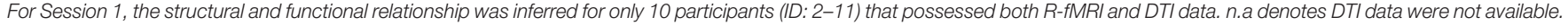

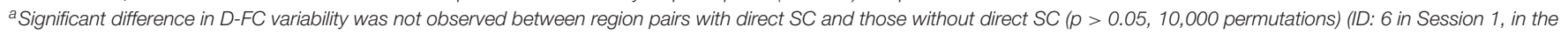
AAL-90 brain networks).

${ }^{b} D$-FC variability did not exhibit significant correlation with SC strength $(p>0.05)$. 
TABLE 3 | Summary of global properties of individual structural brain networks (AAL-90).

\begin{tabular}{|c|c|c|c|c|c|c|c|}
\hline Participant ID & $S$ & $C_{p}$ & $L_{p}$ & $\gamma$ & $\lambda$ & $\sigma$ & $\alpha$ \\
\hline 1 & n.a. & n.a. & n.a. & n.a. & n.a. & n.a. & n.a. \\
\hline 2 & 0.19 & 0.53 & 1.78 & 2.18 & 1.05 & 2.08 & 0.02 \\
\hline 3 & 0.19 & 0.54 & 1.86 & 2.41 & 1.09 & 2.21 & 0.06 \\
\hline 4 & 0.21 & 0.55 & 1.77 & 2.06 & 1.06 & 1.95 & 0.02 \\
\hline 5 & 0.22 & 0.52 & 1.72 & 1.90 & 1.04 & 1.83 & 0.04 \\
\hline 6 & 0.22 & 0.58 & 1.76 & 2.12 & 1.06 & 1.99 & 0.02 \\
\hline 7 & 0.22 & 0.54 & 1.75 & 2.05 & 1.05 & 1.95 & 0.06 \\
\hline 8 & 0.20 & 0.55 & 1.81 & 2.26 & 1.07 & 2.11 & 0.01 \\
\hline 9 & 0.20 & 0.55 & 1.78 & 2.15 & 1.06 & 2.03 & 0.04 \\
\hline 10 & 0.22 & 0.56 & 1.74 & 2.03 & 1.05 & 1.92 & 0.01 \\
\hline 11 & 0.21 & 0.54 & 1.73 & 1.82 & 1.04 & 1.75 & 0.03 \\
\hline Mean \pm std & $0.21 \pm 0.01$ & $0.55 \pm 0.02$ & $1.77 \pm 0.04$ & $2.10 \pm 0.17$ & $1.06 \pm 0.01$ & $1.98 \pm 0.13$ & $0.03 \pm 0.02$ \\
\hline
\end{tabular}

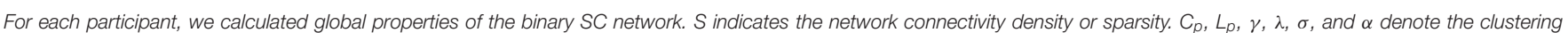

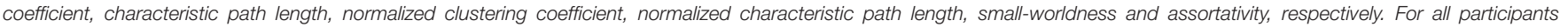

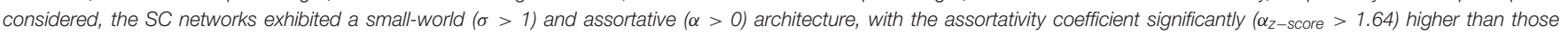
in random networks. Notably, only 10 participants (ID: 2-11) that possessed DTI data were included in structural network analysis. n.a. denotes DTI data were not available.

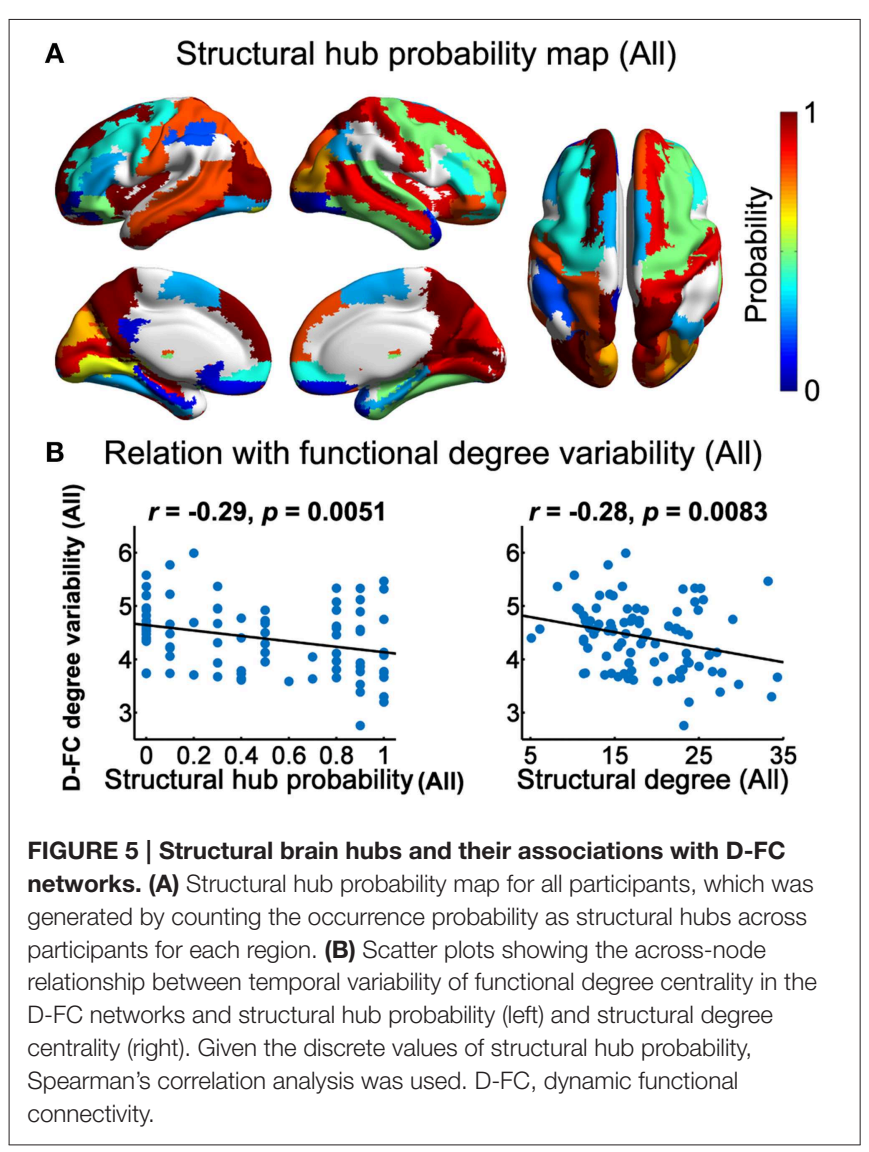

The effects of different scanning session. We found that most of the findings reported above were highly reproducible using the multiband R-fMRI and DTI data obtained from the same participants during the second scanning session, which was separately from the first by approximately 1 week
(Table 2, Figure S1). (ii) The effects of window length. We regenerated the individual D-FC networks using different window lengths (window length $=50$ or $150 \mathrm{~s}$ ) and found that the temporal features of the functional organization, including the connections, global properties and nodal degree, were highly similar to our main results (Figures S2, S3). Moreover, the connectivity strength and temporal variability of the D-FC were significantly correlated with SC strength at different window lengths, but the relationship was more significant when a smaller window length was used (Figure S2D vs. Figure S3D). (iii) The effects of correlation thresholding strategy. We found that the main results were preserved when different correlation thresholding strategies were used for D-FC network construction, including the varying Bonferroni correction thresholds $\left(p_{c o r r}<0.05\right.$ or 0.001 ; Figures $S 4, S 5)$, and different network sparsity values $(S=$ 10, 15, and 20\%; Figure S6). (iv) The effects of network type. We regenerated weighted D-FC networks, and found that retaining the edge weights during the brain network analyses did not change our main findings (Figure S7).

\section{Discussion}

Using the sub-second multiband R-fMRI and DTI data, we comprehensively explored the dynamic topological organization of human whole-brain functional networks at a finer temporal resolution ( $\mathrm{TR}=645 \mathrm{~ms}$ ) and provided the first demonstration of their underlying structural substrates. The main results were summarized as follows: (i) The overall D-FC patterns maintained high spatial similarity with the static connectivity pattern, with larger connectivity strength and lower temporal variability in homotopic D-FC than heterotopic and intrahemispheric DFC. The D-FC networks exhibited time-varying but persistent small-worldness, assortativity and functional hubs, with several regions (e.g., insula, sensorimotor cortex, and medial prefrontal cortex) emerging as functionally persistent hubs but having larger temporal variability in degree centrality than other regions. (ii) 
TABLE 4 | Structurally persistent brain hubs across participants (AAL-90).

\begin{tabular}{|c|c|c|}
\hline ROI name & $\begin{array}{c}\text { Hub } \\
\text { probability }\end{array}$ & $\begin{array}{c}\text { Structural } \\
\text { degree centrality }\end{array}$ \\
\hline L Superior frontal gyrus, dorsolateral & 1.00 & 27.10 \\
\hline L Superior frontal gyrus, medial & 1.00 & 26.60 \\
\hline L Insula & 1.00 & 23.10 \\
\hline R Calcarine fissure and surrounding cortex & 1.00 & 23.80 \\
\hline L Middle occipital gyrus & 1.00 & 27.70 \\
\hline R Superior parietal gyrus & 1.00 & 26.20 \\
\hline L Precuneus & 1.00 & 34.30 \\
\hline R Precuneus & 1.00 & 33.60 \\
\hline L Lenticular nucleus, putamen & 1.00 & 29.00 \\
\hline R Lenticular nucleus, putamen & 1.00 & 33.20 \\
\hline R Superior frontal gyrus, dorsolateral & 0.90 & 29.70 \\
\hline L Superior frontal gyrus, orbital part & 0.90 & 23.80 \\
\hline R Insula & 0.90 & 25.20 \\
\hline L Hippocampus & 0.90 & 25.50 \\
\hline L Calcarine fissure and surrounding cortex & 0.90 & 23.20 \\
\hline R Cuneus & 0.90 & 25.00 \\
\hline R Lingual gyrus & 0.90 & 27.50 \\
\hline R Postcentral gyrus & 0.90 & 22.90 \\
\hline R Caudate nucleus & 0.90 & 22.40 \\
\hline R Middle temporal gyrus & 0.90 & 22.20 \\
\hline L Inferior temporal gyrus & 0.90 & 22.70 \\
\hline R Superior frontal gyrus, orbital part & 0.80 & 23.60 \\
\hline R Inferior frontal gyrus, orbital part & 0.80 & 24.50 \\
\hline R Superior frontal gyrus, medial & 0.80 & 22.80 \\
\hline L Superior occipital gyrus & 0.80 & 23.10 \\
\hline R Superior occipital gyrus & 0.80 & 23.70 \\
\hline L Postcentral gyrus & 0.80 & 21.40 \\
\hline L Superior parietal gyrus & 0.80 & 23.50 \\
\hline L Caudate nucleus & 0.80 & 24.50 \\
\hline L Thalamus & 0.80 & 24.90 \\
\hline L Middle temporal gyrus & 0.80 & 22.30 \\
\hline L Cuneus & 0.70 & 21.80 \\
\hline R Middle occipital gyrus & 0.70 & 20.80 \\
\hline L Lingual gyrus & 0.60 & 20.10 \\
\hline
\end{tabular}

Persistent structural hub regions across participants (i.e., probability $>0.5$ ) are listed here. $L$, left; $R$, right. Hub probability represents the occurrence probability as structural hubs across participants, and structural degree centrality denotes the average nodal structural degree values across participants.

The temporal characteristics (i.e., strength and variability) of the D-FC were significantly associated with the existence and the strength of structural connections. The temporal variability of nodal degree centrality in the D-FC networks was anatomically constrained by the structural networks. Collectively, these results provide novel insights into the spontaneous or intrinsic network dynamics of the human brain and their underlying structural substrates at a finer time scale (seconds).

\section{Dynamic Functional Connectivity Patterns in the Resting Human Brain}

Traditional static FC analysis using the whole-scan time courses has greatly advanced our understanding of the intrinsic functional organization of the brain (Biswal et al., 1995; Fox and Raichle, 2007; Kelly et al., 2012). However, this approach cannot capture the time-varying characteristics of functional organization. Using a sliding window approach, in the present study, we observed that functional interactions among regions during rest underwent spontaneous dynamic reorganization (Figure 1, Videos S1, S3). On the one hand, these diverse DFC patterns may be due to the shift of arousal states during the unconstrained resting scanning (Allen et al., 2014). On the other hand, and more possibly, the spontaneous fluctuations of D-FC patterns may be the manifestation of intrinsic brain dynamics (Hutchison et al., 2013b). Recent empirical (Tagliazucchi et al., 2012a; Liu and Duyn, 2013) and theoretical (Deco et al., 2011, 2013; Haimovici et al., 2013) studies suggest that the resting brain may reside near a critical state, supporting a rich repertoire of functional coordination patterns. Thus, the switch of largescale D-FC patterns may reflect the exploration of available functional configurations, which would benefit the efficiency and the speed for the configuration selection in response to potential cognitive demands (Deco et al., 2013). Interestingly, previous electrophysiological studies (Kramer et al., 2011; Chu et al., 2012) with long-term recordings ( $\sim$ days) in the human brain found that a network template emerged from short time intervals $(\sim$ $100 \mathrm{~s})$ underlying the dynamic functional organization. Here, we provide evidence that the large-scale D-FC patterns derived from R-fMRI data persistently exhibited high spatial similarity with the static pattern (Figures 1C, 6A), indicating that the latter might serve as a template on which diverse, transient functional configurations emerge and recede with minor modulations. We assume that the template for functional coordination may be manifestation of the influence of anatomical architecture, the detection and the physiological meaning of which require further elucidation.

\section{Dynamic Small-World and Assortative Functional Brain Networks}

Small-world brain networks serve as an attractive model for brain organization, because they supports efficient information segregation and integration through dense local clustering and yet short path length between any pair of nodes (Watts and Strogatz, 1998). A number of studies have consistently found small-world architecture in static functional brain networks across diverse imaging modalities (e.g., fMRI, EEG, and MEG) (for reviews, see Bassett and Bullmore, 2006; Bullmore and Sporns, 2009, 2012; He and Evans, 2010), suggesting that it is a general principle of brain organization. Using a sliding window approach, we observed that although the measure of small-worldness fluctuated substantially over time, smallworld topology was preserved across all of the windows in company with low sparsity (i.e., wiring costs; Figures 2, 6B), indicating that the human brain maintains a dynamic balance between efficient local and global communications to meet changeable environments. More specifically, although the functional connections attached to each node were variable across time (Figure 3A), the functional connection patterns across the whole brain maintained significant assortativity (Figures 2, 6B). As a consequence, high-degree hub nodes 
A
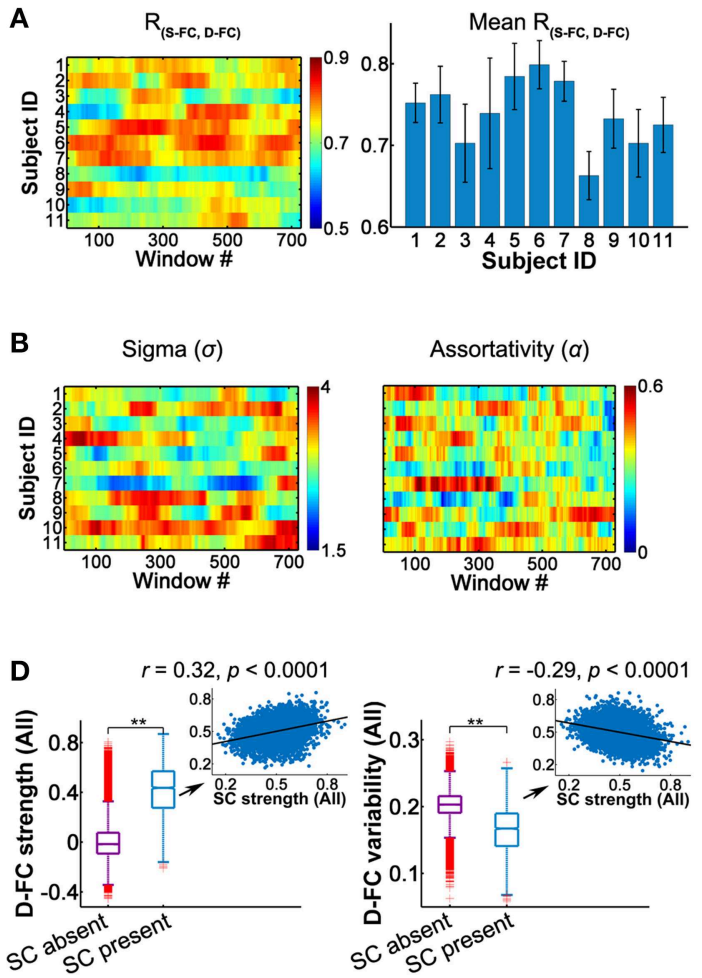
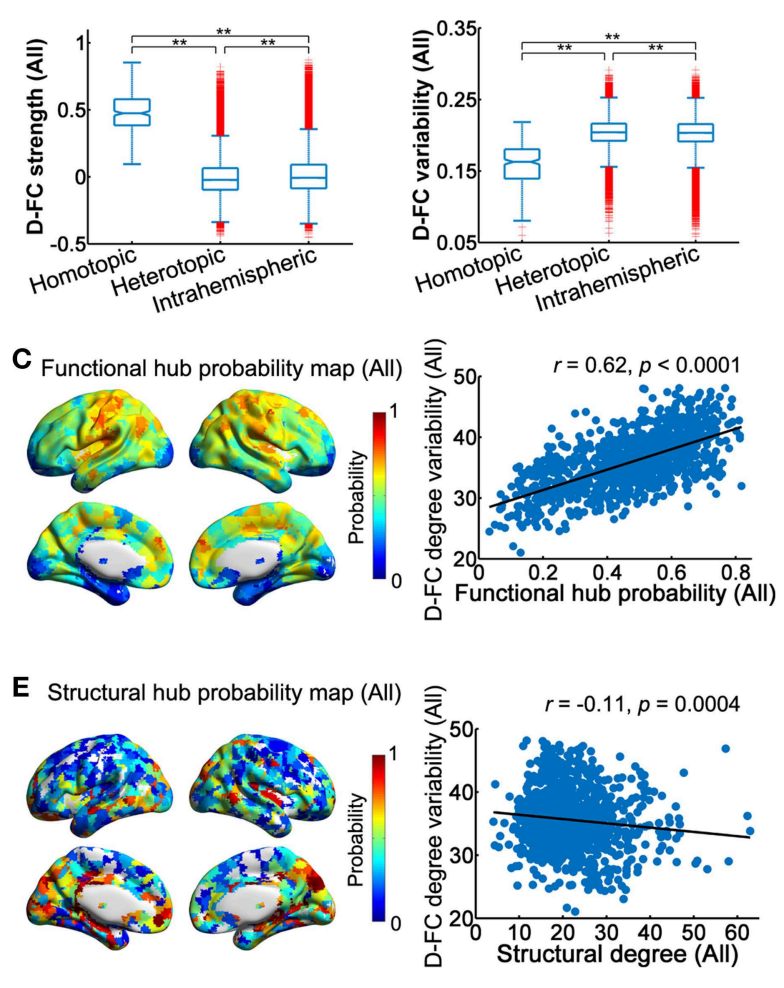

FIGURE 6 | Dynamic characteristics of high-resolution (H-1024) D-FC brain networks and their structural associations. (A) Spatial similarity between the S-FC and the D-FC matrices over time (left) and the spatial-dependence of D-FC strength and variability for all participants (right). ${ }^{* *}$ Bonferroni corrected $p<0.001$, 10,000 permutations. (B) Time-varying small-worldness and assortativity of D-FC networks. (C) Functional hub probability map for all participants (left) and scatter plot showing across-node relationship between functional hub probability and the temporal variability of functional degree centrality in the D-FC networks (right). (D) Differences in D-FC strength (left) and temporal variability (right) between region pairs with or without direct SC. ${ }^{\star *} p<0.0001$. Scatter plots (insets) represent the across-connection relationship of D-FC strength (left) and D-FC variability (right) vs. SC strength, confined to the regional pairs with direct SC. Relevant statistical analyses were performed for all participants based on the SC backbone network. (E) Structural hub probability map for all participants (left) and across-node relationship with the temporal variability of functional degree centrality (right). The hub map was generated by counting the occurrence probability as structural hubs across participants for each region. SC, structural connectivity; S-FC, static functional connectivity; D-FC, dynamic functional connectivity.

preferentially connected with each other, constituting a relatively resilient "connectivity core." Assortative topology has been readily observed in static functional networks of the human brain (Eguíluz et al., 2005; Park et al., 2008; Braun et al., 2012). Maintaining this feature during dynamic organization could be beneficial for continual information processing among hubs, and could thus promote a fast response to changing cognitive demands and increase the resilience to potential pathological attacks.

\section{Persistent but Flexible Functional Brain Hubs}

We found that brain regions exhibited substantial fluctuations across time in their nodal degree centrality values (Figure 3A, Videos S2, S4), indicating their variable functional roles at a short time scale. Of note, several regions emerged as functionally persistent hubs (i.e., $>50 \%$ of the windows) over time (Figures 3B, 6C), which were mainly located in the sensorimotor cortex, default-mode network (e.g., medial prefrontal cortex, angular gyrus, posterior cingulate gyrus and precuneus) and the cingulo-opercular control system (e.g., anterior cingulate cortex and insula). The majority of these regions have been identified as functional hubs in previous static network studies (Achard et al., 2006; Buckner et al., 2009; Liang et al., 2013; Liao et al., 2013; van den Heuvel and Sporns, 2013) and are supposed to be crucial for efficient functional coordination across spatially distributed regions. Notably, our findings highlight their enduring contributions to global information integration and provide insights into their functional roles in dynamic network integrity. Interestingly, previous studies of the human brain (Allen et al., 2014; Zalesky et al., 2014) suggest that functional connectivity involving these regions is highly variable over time. Here, using quantitative degree centrality analysis, we demonstrated that the functionally persistent hubs tended to exhibit larger temporal variability in functional degree centrality than other regions, regardless of the spatial resolution considered (Figures 3B, 6C). Interestingly, highly variable functional hubs have also been observed in macaque cortical networks using both simulation (Honey et al., 2007) and empirical (Shen et al., 2015) data, in which functional hubs were estimated from longer time periods ( $\sim$ minutes). In a task-based fMRI study, Cole 
et al. (2013) pointed out that the flexible fronto-parietal hub regions may update their functional connectivity patterns rapidly according to different task states. Using multiband R-fMRI, we further demonstrated the dynamic characteristics of functional brain hubs during rest, including their persistence and flexibility across time. These persistent hubs dynamically adjust their global functional connections involving multiple systems, to enable adaptive information exchange and integration.

\section{Structural Substrates of Dynamic Functional Organization}

Simulations of macaque brain networks have shown that interregional functional connectivity at multiple time scales (e.g., seconds to minutes) is shaped by the anatomical architecture (Honey et al., 2007). Very recently, using R-fMRI in macaques Shen et al. (2015) provided an empirical demonstration that more stable functional connections are observed between region pairs with direct anatomical connections obtained from axonal tract tracing. However, for the human brain, the majority of studies have focused mainly on the structural substrates for static functional connectivity or networks, and the structural constraints on spontaneous evolution of functional networks are still unclear. Based on multiband R-fMRI and DTI data of the same participants, we provided crucial evidence for the structural substrates underlying the large-scale dynamic functional organization of the human brain on the order of seconds, at both connection and network levels.

At the connection level, we found that both the connectivity strength and the temporal variability of D-FC were dependent on the spatial interrelationships (Figures 1D, 6A), which agrees well with previous studies on S-FC (Stark et al., 2008) and D-FC that fluctuates on a scale of minutes (Gonzalez-Castillo et al., 2014). One possible reason is that as homologous regions possess similar functional roles (Toro et al., 2008; Crossley et al., 2013), homotopic FC between them may manifest as continual functional interaction, with less temporal variation. In contrast, heterotopic and intrahemispheric FC usually connects regions involved in specialized functions, yielding occasional interregional functional coupling with larger temporal variability. Moreover, the direct SC, corpus callosum, linking homologous regions (Gong et al., 2009; van den Heuvel et al., 2009) may promote the temporal stability of homotopic FC. Although prior studies have shown that SC may shape the functional interactions or S-FC (Hagmann et al., 2008; Honey et al., 2009; van den Heuvel et al., 2009; Goñi et al., 2014), the structural influences on the temporal characteristics of functional couplings are usually ignored. Here, we highlighted that D-FC fluctuating on a scale of seconds was shaped by the underlying SC: the larger the structural connectivity strength, the greater the functional coupling with less temporal variability (Figures 4, 6D and Table 2), which extends our understanding of the structurefunction relationships in the human brain at shorter time scales.

At the network level, we found that the dynamic functional networks and structural networks shared common global properties (e.g., small-worldness and assortativity; Figure 2 and Table 3), which was compatible with prior static FC studies (Bullmore and Sporns, 2009, 2012). Moreover, we observed that regions that possessed larger structural degree centrality or structural hub probability tended to exhibit less temporal variability of functional degree centrality, which could be due to the structural constraints on temporal variability of $\mathrm{D}$ FC (Figure 4), as mentioned above. Of note, this negative relationship between structural degree centrality and the temporal variability of functional degree centrality is different from that for functional hubs, which may result from the different spatial patterns of the probability maps observed for functional hubs (Figure 3B) and structural hubs (Figure 5A). Although several hub regions in common (e.g., insula, medial prefrontal cortex) were found, we also observed that several structural hub regions (e.g., middle temporal gyrus and visual cortex) exhibited small probability as functional hubs, and several functionally persistent hubs (e.g., anterior cingulate cortex) possessed small structural degree centrality. These discrepancies in nodal centrality may be partially attributed to the fact that strong D-FC can also exist between region pairs without direct SC, as illustrated by the red outliers in Figure 4B (middle panel). As suggested by several static FC studies (Honey et al., 2009; Adachi et al., 2012), this unexpected D-FC could be mediated by indirect SC, making the inference of functionally persistent hubs from structural hubs via one-to-one mapping unfeasible. Additionally, the estimation of global network topology involves the large-scale connectivity patterns across the whole brain; thus the quantitative correspondence between structural and dynamic functional networks becomes difficult.

Recently, based on empirical SC data, several computational model studies point out that not only the anatomical structure but also the dynamics of local regions can affect the inter-regional functional coupling (Honey et al., 2009; Deco and Jirsa, 2012; Deco et al., 2013; Hansen et al., 2015). Through an enhanced non-linear mean-field model, Hansen et al. (2015) simulated the brain activities at a subcritical regime, and reproduced the spontaneous switches of FC states. These studies suggest that the behavior of D-FC cannot be understood in terms of SC alone, and incorporating computational models in future studies can obtain mechanistic insights into the complex structure-function relationship in the brain.

\section{Further Considerations}

Several issues should be considered further. First, the limited number of participants used for this study (11 participants) may restrict the statistical power of the results. Although the main results were replicated here by using repeated dataset (two sessions) of the same participants acquired around 1 week apart, it would be worthy to replicate the present findings based on a larger dataset in the future. Second, to capture the dynamics of functional networks, a commonly used sliding window approach was used here. However, to date, there is no universally accepted criterion for window selection (Hutchison et al., 2013a). To ensure the robustness of our results, three window lengths, 50, 100, and $150 \mathrm{~s}$, were considered in the current study. Because, increasing attention has been paid to the time-varying functional organization of the brain (Hutchison et al., 2013a; Calhoun et al., 2014), a methodological framework 
for the choice of more appropriate windows (e.g., window type and window length) should be established with future efforts. Third, we found some differences across different parcellation schemes considered, such as additional functional hubs in the posterior cingulate cortex and precuneus in the high-resolution (H-1024) networks. These discrepancies could be due to that some specific ROIs (e.g., precuneus) in the AAL-90 parcellation are functionally inhomogeneous. Thus, the potential influence of different parcellation schemes on evaluating functional network dynamics deserves further investigation. Fourth, previous studies have suggested that head motion may introduce artificial functional connections (Power et al., 2012; Van Dijk et al., 2012; Satterthwaite et al., 2013). Several motion correction strategies have been recently proposed, including the widely used scrubbing approach (Power et al., 2012). Notably, data scrubbing involves the removal or interpolation of contaminated time points, which may damage the temporal continuity of time courses and thereby affect the estimation of D-FC patterns. Therefore, in the present study, we used a 24-parameter autoregression model (Friston et al., 1996) during data preprocessing to partially reduce the motion-induced artifacts while maintaining the temporal continuity of data. Future studies of developing new motion correction strategies are important to evaluate the potential influence of head motion on dynamic functional networks. Fifth, we employed a subsecond $(\mathrm{TR}=645 \mathrm{~ms}$ ) multiband R-fMRI technique for brain network dynamics, which captures fine temporal information of BOLD activities and partially removes the influence of

\section{References}

Achard, S., Salvador, R., Whitcher, B., Suckling, J., and Bullmore, E. (2006). A resilient, low-frequency, small-world human brain functional network with highly connected association cortical hubs. J. Neurosci. 26, 63-72. doi: 10.1523/JNEUROSCI.3874-05.2006

Adachi, Y., Osada, T., Sporns, O., Watanabe, T., Matsui, T., Miyamoto, K., et al. (2012). Functional connectivity between anatomically unconnected areas is shaped by collective network-level effects in the macaque cortex. Cereb. Cortex 22, 1586-1592. doi: 10.1093/cercor/bhr234

Allen, E. A., Damaraju, E., Plis, S. M., Erhardt, E. B., Eichele, T., and Calhoun, V. D. (2014). Tracking whole-brain connectivity dynamics in the resting state. Cereb. Cortex 24, 663-676. doi: 10.1093/cercor/bhs352

Ashburner, J., and Friston, K. J. (1999). Nonlinear spatial normalization using basis functions. Hum. Brain Mapp. 7, 254-266.

Basser, P., and Pierpaoli, C. (1996). Microstructural and physiological features of tissues elucidated by quantitative-diffusion-tensor MRI. J. Magn. Reson. B 111, 209-219. doi: 10.1006/jmrb.1996.0086

Basser, P. J., Mattiello, J., and LeBihan, D. (1994). MR diffusion tensor spectroscopy and imaging. Biophys. J. 66, 259. doi: 10.1016/S0006-3495(94)80775-1

Bassett, D. S., and Bullmore, E. (2006). Small-world brain networks. Neuroscientist 12, 512-523. doi: $10.1177 / 1073858406293182$

Biswal, B., Yetkin, F. Z., Haughton, V. M., and Hyde, J. S. (1995). Functional connectivity in the motor cortex of resting human brain using echo-planar MRI. Magn. Reson. Med. 34, 537-541. doi: 10.1002/mrm.1910340409

Braun, U., Plichta, M. M., Esslinger, C., Sauer, C., Haddad, L., Grimm, O., et al. (2012). Test-retest reliability of resting-state connectivity network characteristics using fMRI and graph theoretical measures. Neuroimage 59, 1404-1412. doi: 10.1016/j.neuroimage.2011.08.044

Buckner, R. L., Sepulcre, J., Talukdar, T., Krienen, F. M., Liu, H., Hedden, T., et al. (2009). Cortical hubs revealed by intrinsic functional connectivity: mapping, respiratory signals $(\sim 0.3 \mathrm{~Hz})$. Further works using advanced imaging protocols with higher sampling rates and simultaneous physiological signal recordings would be important to better infer the brain dynamics and further diminish the effects of non-neural signals (e.g., cardiac rhythms). Finally, we showed the dynamic features of functional brain networks and their structural correlates. These patterns could be modulated by different cognitive tasks and during normal development, aging and neuropsychiatric disorders, all of which are of interested for future studies.

\section{Acknowledgments}

This work was supported by the National Key Basic Research Program of China (Grant Nos. 2014CB846102 and 2013CB837300), the Natural Science Foundation of China (Grant Nos. 11205041, 81401479, 91432115, and 31221003), the National Science Fund for Distinguished Young Scholars (Grant No. 81225012), Beijing Natural Science Foundation (Grant No. Z151100003915082) and the Open Research Fund of the State Key Laboratory of Cognitive Neuroscience and Learning (CNLYB1307).

\section{Supplementary Material}

The Supplementary Material for this article can be found online at: http://journal.frontiersin.org/article/10.3389/fnhum. 2015.00478 assessment of stability, and relation to Alzheimer's disease. J. Neurosci. 29, 1860-1873. doi: 10.1523/JNEUROSCI.5062-08.2009

Bullmore, E., and Sporns, O. (2009). Complex brain networks: graph theoretical analysis of structural and functional systems. Nat. Rev. Neurosci. 10, 186-198. doi: $10.1038 / \mathrm{nrn} 2575$

Bullmore, E., and Sporns, O. (2012). The economy of brain network organization. Nat. Rev. Neurosci. 13, 336-349. doi: 10.1038/nrn3214

Calhoun, V. D., Miller, R., Pearlson, G., and Adali, T. (2014). The chronnectome: time-varying connectivity networks as the next frontier in fMRI data discovery. Neuron 84, 262-274. doi: 10.1016/j.neuron. 2014.10.015

Chang, C., and Glover, G. H. (2010). Time-frequency dynamics of restingstate brain connectivity measured with fMRI. Neuroimage 50, 81-98. doi: 10.1016/j.neuroimage.2009.12.011

Chang, C., Liu, Z. M., Chen, M. C., Liu, X., and Duyn, J. H. (2013). EEG correlates of time-varying BOLD functional connectivity. Neuroimage 72, 227-236. doi: 10.1016/j.neuroimage.2013.01.049

Chu, C. J., Kramer, M. A., Pathmanathan, J., Bianchi, M. T., Westover, M. B., Wizon, L., et al. (2012). Emergence of stable functional networks in long-term human electroencephalography. J. Neurosci. 32, 2703-2713. doi: 10.1523/JNEUROSCI.5669-11.2012

Cole, M. W., Reynolds, J. R., Power, J. D., Repovs, G., Anticevic, A., and Braver, T. S. (2013). Multi-task connectivity reveals flexible hubs for adaptive task control. Nat. Neurosci. 16, 1348-1355. doi: 10.1038/nn.3470

Crossley, N. A., Mechelli, A., Vertes, P. E., Winton-Brown, T. T., Patel, A. X., Ginestet, C. E., et al. (2013). Cognitive relevance of the community structure of the human brain functional coactivation network. Proc. Natl. Acad. Sci. U.S.A. 110, 11583-11588. doi: 10.1073/pnas.1220826110

Deco, G., and Jirsa, V. K. (2012). Ongoing cortical activity at rest: criticality, multistability, and ghost attractors. J. Neurosci. 32, 3366-3375. doi: 10.1523/JNEUROSCI.2523-11.2012 
Deco, G., Jirsa, V. K., and McIntosh, A. R. (2011). Emerging concepts for the dynamical organization of resting-state activity in the brain. Nat. Rev. Neurosci. 12, 43-56. doi: 10.1038/nrn2961

Deco, G., Jirsa, V. K., and McIntosh, A. R. (2013). Resting brains never rest: computational insights into potential cognitive architectures. Trends Neurosci. 36, 268-274. doi: 10.1016/j.tins.2013.03.001

Di, X., and Biswal, B. B. (2015). Dynamic brain functional connectivity modulated by resting-state networks. Brain Struct. Funct. 220, 37-46. doi: 10.1007/s00429013-0634-3

Eguíluz, V. M., Chialvo, D. R., Cecchi, G. A., Baliki, M., and Apkarian, A. V. (2005). Scale-free brain functional networks. Phys. Rev. Lett. 94:018102. doi: 10.1103/PhysRevLett.94.018102

Fornito, A., Zalesky, A., and Bullmore, E. T. (2010). Network scaling effects in graph analytic studies of human resting-state FMRI data. Front. Syst. Neurosci. 4:22. doi: 10.3389/fnsys.2010.00022

Fox, M. D., and Raichle, M. E. (2007). Spontaneous fluctuations in brain activity observed with functional magnetic resonance imaging. Nat. Rev. Neurosci. 8, 700-711. doi: 10.1038/nrn2201

Fox, M. D., Snyder, A. Z., Vincent, J. L., Corbetta, M., Van Essen, D. C., and Raichle, M. E. (2005). The human brain is intrinsically organized into dynamic, anticorrelated functional networks. Proc. Natl. Acad. Sci. U.S.A. 102, 9673-9678. doi: 10.1073/pnas.0504136102

Fox, M. D., Zhang, D., Snyder, A. Z., and Raichle, M. E. (2009). The global signal and observed anticorrelated resting state brain networks. J. Neurophysiol. 101, 3270-3283. doi: 10.1152/jn.90777.2008

Friston, K. J., Williams, S., Howard, R., Frackowiak, R. S., and Turner, R. (1996). Movement-related effects in fMRI time-series. Magn. Reson. Med. 35, 346-355. doi: 10.1002/mrm.1910350312

Gong, G., He, Y., Concha, L., Lebel, C., Gross, D. W., Evans, A. C., et al. (2009). Mapping anatomical connectivity patterns of human cerebral cortex using in vivo diffusion tensor imaging tractography. Cereb. Cortex 19, 524-536. doi: $10.1093 /$ cercor/bhn 102

Goñi, J., van den Heuvel, M. P., Avena-Koenigsberger, A., de Mendizabal, N. V., Betzel, R. F., Griffa, A., et al. (2014). Resting-brain functional connectivity predicted by analytic measures of network communication. Proc. Natl. Acad. Sci. U.S.A. 111, 833-838. doi: 10.1073/pnas.1315529111

Gonzalez-Castillo, J., Handwerker, D. A., Robinson, M. E., Hoy, C. W., Buchanan, L. C., Saad, Z. S., et al. (2014). The spatial structure of resting state connectivity stability on the scale of minutes. Front. Neurosci. 8:138. doi: 10.3389/fnins.2014.00138

Greicius, M. D., Supekar, K., Menon, V., and Dougherty, R. F. (2009). Restingstate functional connectivity reflects structural connectivity in the default mode network. Cereb. Cortex 19, 72-78. doi: 10.1093/cercor/bhn059

Hagmann, P., Cammoun, L., Gigandet, X., Meuli, R., Honey, C. J., Wedeen, V., et al. (2008). Mapping the structural core of human cerebral cortex. PLoS Biol. 6:e159. doi: 10.1371/journal.pbio.0060159

Haimovici, A., Tagliazucchi, E., Balenzuela, P., and Chialvo, D. R. (2013). Brain organization into resting state networks emerges at criticality on a model of the human connectome. Phys. Rev. Lett. 110:178101. doi: 10.1103/PhysRevLett.110.178101

Hansen, E. C., Battaglia, D., Spiegler, A., Deco, G., and Jirsa, V. K. (2015). Functional connectivity dynamics: modeling the switching behavior of the resting state. Neuroimage 105, 525-535. doi: 10.1016/j.neuroimage.2014.11.001

He, Y., and Evans, A. (2010). Graph theoretical modeling of brain connectivity. Curr. Opin. Neurol. 23, 341-350. doi: 10.1097/wco.0b013e32833aa567

Honey, C. J., Kötter, R., Breakspear, M., and Sporns, O. (2007). Network structure of cerebral cortex shapes functional connectivity on multiple time scales. Proc. Natl. Acad. Sci. U.S.A. 104, 10240-10245. doi: 10.1073/pnas. 0701519104

Honey, C. J., Sporns, O., Cammoun, L., Gigandet, X., Thiran, J. P., Meuli, R., et al. (2009). Predicting human resting-state functional connectivity from structural connectivity. Proc. Natl. Acad. Sci. U.S.A. 106, 2035-2040. doi: 10.1073/pnas.0811168106

Humphries, M. D., and Gurney, K. (2008). Network 'small-world-ness': a quantitative method for determining canonical network equivalence. PLoS ONE 3:e0002051. doi: 10.1371/journal.pone.0002051

Hutchison, R. M., Womelsdorf, T., Allen, E. A., Bandettini, P. A., Calhoun, V. D., Corbetta, M., et al. (2013a). Dynamic functional connectivity: promise, issues, and interpretations. Neuroimage 80, 360-378. doi: 10.1016/j.neuroimage.2013.05.079

Hutchison, R. M., Womelsdorf, T., Gati, J. S., Everling, S., and Menon, R. S. (2013b). Resting-state networks show dynamic functional connectivity in awake humans and anesthetized macaques. Hum. Brain Mapp. 34, 2154-2177. doi: 10.1002/hbm.22058

Jones, D. T., Vemuri, P., Murphy, M. C., Gunter, J. L., Senjem, M. L., Machulda, M. M., et al. (2012). Non-stationarity in the "resting brain's" modular architecture. PLoS ONE 7:e39731. doi: 10.1371/journal.pone.0039731

Kang, J., Wang, L., Yan, C. G., Wang, J. H., Liang, X., and He, Y. (2011). Characterizing dynamic functional connectivity in the resting brain using variable parameter regression and Kalman filtering approaches. Neuroimage 56, 1222-1234. doi: 10.1016/j.neuroimage.2011.03.033

Kelly, C., Biswal, B. B., Craddock, R. C., Castellanos, F. X., and Milham, M. P. (2012). Characterizing variation in the functional connectome: promise and pitfalls. Trends Cogn. Sci. 16, 181-188. doi: 10.1016/j.tics.2012.02.001

Kiviniemi, V., Vire, T., Remes, J., Elseoud, A. A., Starck, T., Tervonen, O. et al. (2011). A sliding time-window ICA reveals spatial variability of the default mode network in time. Brain Connect. 1, 339-347. doi: 10.1089/brain 2011.0036

Kramer, M. A., Eden, U. T., Lepage, K. Q., Kolaczyk, E. D., Bianchi, M. T., and Cash, S. S. (2011). Emergence of persistent networks in long-term intracranial EEG recordings. J. Neurosci. 31, 15757-15767. doi: 10.1523/JNEUROSCI.228711.2011

Li, X., Zhu, D., Jiang, X., Jin, C., Zhang, X., Guo, L., et al. (2014). Dynamic functional connectomics signatures for characterization and differentiation of PTSD patients. Hum. Brain Mapp. 35, 1761-1778. doi: 10.1002/hbm.22290

Liang, X., Zou, Q., He, Y., and Yang, Y. (2013). Coupling of functional connectivity and regional cerebral blood flow reveals a physiological basis for network hubs of the human brain. Proc. Natl. Acad. Sci. U.S.A. 110, 1929-1934. doi: 10.1073/pnas. 1214900110

Liao, X., Xia, M., Xu, T., Dai, Z., Cao, X., Niu, H., et al. (2013). Functional brain hubs and their test-retest reliability: a multiband resting-state functional MRI study. Neuroimage 83, 969-982. doi: 10.1016/j.neuroimage.2013.07.058

Liu, X., and Duyn, J. H. (2013). Time-varying functional network information extracted from brief instances of spontaneous brain activity. Proc. Natl. Acad. Sci. U.S.A. 110, 4392-4397. doi: 10.1073/pnas.1216856110

Maslov, S., and Sneppen, K. (2002). Specificity and stability in topology of protein networks. Science 296, 910-913. doi: 10.1126/science.1065103

Mori, S., Crain, B. J., Chacko, V., and Van Zijl, P. (1999). Three-dimensional tracking of axonal projections in the brain by magnetic resonance imaging. Ann. Neurol. 45, 265-269.

Murphy, K., Birn, R. M., Handwerker, D. A., Jones, T. B., and Bandettini, P. A. (2009). The impact of global signal regression on resting state correlations: are anti-correlated networks introduced? Neuroimage 44, 893-905. doi: 10.1016/j.neuroimage.2008.09.036

Newman, M. E. (2002). Assortative mixing in networks. Phys. Rev. Lett. 89:208701. doi: 10.1103/PhysRevLett.89.208701

Nooner, K. B., Colcombe, S. J., Tobe, R. H., Mennes, M., Benedict, M. M., Moreno, A. L., et al. (2012). The NKI-Rockland sample: a model for accelerating the pace of discovery science in psychiatry. Front. Neurosci. 6:152. doi: 10.3389/fnins.2012.00152

Park, C.-H., Kim, S. Y., Kim, Y.-H., and Kim, K. (2008). Comparison of the smallworld topology between anatomical and functional connectivity in the human brain. Phys. A 387, 5958-5962. doi: 10.1016/j.physa.2008.06.048

Park, H.-J., and Friston, K. (2013). Structural and functional brain networks: from connections to cognition. Science 342:1238411. doi: 10.1126/science.1238411

Power, J. D., Barnes, K. A., Snyder, A. Z., Schlaggar, B. L., and Petersen, S. E. (2012). Spurious but systematic correlations in functional connectivity MRI networks arise from subject motion. Neuroimage 59, 2142-2154. doi: 10.1016/j.neuroimage.2011.10.018

Rubinov, M., and Sporns, O. (2010). Complex network measures of brain connectivity: uses and interpretations. Neuroimage 52, 1059-1069. doi: 10.1016/j.neuroimage.2009.10.003

Salvador, R., Suckling, J., Coleman, M. R., Pickard, J. D., Menon, D., and Bullmore, E. (2005). Neurophysiological architecture of functional magnetic resonance images of human brain. Cereb. Cortex 15, 1332-1342. doi: 10.1093/cercor/bhi016 
Satterthwaite, T. D., Elliott, M. A., Gerraty, R. T., Ruparel, K., Loughead, J., Calkins, M. E., et al. (2013). An improved framework for confound regression and filtering for control of motion artifact in the preprocessing of resting-state functional connectivity data. Neuroimage 64, 240-256. doi: 10.1016/j.neuroimage.2012.08.052

Shen, K., Hutchison, R. M., Bezgin, G., Everling, S., and McIntosh, A. R. (2015). Network structure shapes spontaneous functional connectivity dynamics. J. Neurosci. 35, 5579-5588. doi: 10.1523/JNEUROSCI.4903-14.2015

Shu, N., Liu, Y., Li, K., Duan, Y., Wang, J., Yu, C., et al. (2011). Diffusion tensor tractography reveals disrupted topological efficiency in white matter structural networks in multiple sclerosis. Cereb. Cortex 21, 2565-2577. doi: 10.1093/cercor/bhr039

Smith, S. M., Jenkinson, M., Woolrich, M. W., Beckmann, C. F., Behrens, T. E. J., Johansen-Berg, H., et al. (2004). Advances in functional and structural MR image analysis and implementation as FSL. Neuroimage 23, S208-S219. doi: 10.1016/j.neuroimage.2004.07.051

Stark, D. E., Margulies, D. S., Shehzad, Z. E., Reiss, P., Kelly, A. C., Uddin, L. Q., et al. (2008). Regional variation in interhemispheric coordination of intrinsic hemodynamic fluctuations. J. Neurosci. 28, 13754-13764. doi: 10.1523/JNEUROSCI.4544-08.2008

Tagliazucchi, E., Balenzuela, P., Fraiman, D., and Chialvo, D. R. (2012a). Criticality in large-scale brain fMRI dynamics unveiled by a novel point process analysis. Front. Physiol. 3:15. doi: 10.3389/fphys.2012.00015

Tagliazucchi, E., Von Wegner, F., Morzelewski, A., Brodbeck, V., and Laufs, H. (2012b). Dynamic BOLD functional connectivity in humans and its electrophysiological correlates. Front. Hum. Neurosci. 6:339. doi: $10.3389 /$ fnhum.2012.00339

Toro, R., Fox, P. T., and Paus, T. (2008). Functional coactivation map of the human brain. Cereb. Cortex 18, 2553-2559. doi: 10.1093/cercor/ bhn014

Tzourio-Mazoyer, N., Landeau, B., Papathanassiou, D., Crivello, F., Etard, O., Delcroix, N., et al. (2002). Automated anatomical labeling of activations in SPM using a macroscopic anatomical parcellation of the MNI MRI single-subject brain. Neuroimage 15, 273-289. doi: 10.1006/nimg. 2001.0978

van den Heuvel, M. P., Mandl, R. C., Kahn, R. S., and Hulshoff Pol, H. E. (2009). Functionally linked resting-state networks reflect the underlying structural connectivity architecture of the human brain. Hum. Brain Mapp. 30, 3127-3141. doi: 10.1002/hbm.20737

van den Heuvel, M. P., and Sporns, O. (2011). Rich-club organization of the human connectome. J. Neurosci. 31, 15775-15786. doi: 10.1523/JNEUROSCI.353911.2011

van den Heuvel, M. P., and Sporns, O. (2013). Network hubs in the human brain. Trends Cogn. Sci. 17, 683-696. doi: 10.1016/j.tics.2013.09.012

Van Dijk, K. R., Sabuncu, M. R., and Buckner, R. L. (2012). The influence of head motion on intrinsic functional connectivity MRI. Neuroimage 59, 431-438. doi: 10.1016/j.neuroimage.2011.07.044

Wang, J., Wang, L., Zang, Y., Yang, H., Tang, H., Gong, Q., et al. (2009). Parcellation-dependent small-world brain functional networks: A resting-state fMRI study. Hum. Brain Mapp. 30, 1511-1523. doi: 10.1002/hbm.20623
Wang, J., Zuo, X., and He, Y. (2010). Graph-based network analysis of resting-state functional MRI. Front. Syst. Neurosci. 4:16. doi: 10.3389/fnsys.2010.00016

Wang, J. H., Zuo, X. N., Gohel, S., Milham, M. P., Biswal, B. B., and He, Y. (2011). Graph theoretical analysis of functional brain networks: test-retest evaluation on short- and long-term resting-state functional MRI data. PLoS ONE 6:e21976. doi: 10.1371/journal.pone.0021976

Wang, Z., Dai, Z., Gong, G., Zhou, C., and He, Y. (2015). Understanding structuralfunctional relationships in the human brain: a large-scale network perspective. Neuroscientist 21, 290-305. doi: 10.1177/1073858414537560

Watts, D. J., and Strogatz, S. H. (1998). Collective dynamics of 'small-world' networks. Nature 393, 440-442. doi: 10.1038/30918

Weissenbacher, A., Kasess, C., Gerstl, F., Lanzenberger, R., Moser, E., and Windischberger, C. (2009). Correlations and anticorrelations in resting-state functional connectivity MRI: a quantitative comparison of preprocessing strategies. Neuroimage 47, 1408-1416. doi: 10.1016/j.neuroimage.2009.05.005

Wu, G., Liao, W., Stramaglia, S., and Marinazzo, D. (2013). Recovering directed networks in neuroimaging datasets using partially conditioned Granger causality. BMC Neurosci. 14:P260. doi: 10.1089/brain.2013.0142

Xia, M., Wang, J., and He, Y. (2013). BrainNet Viewer: a network visualization tool for human brain connectomics. PLOS ONE 8:e68910. doi: 10.1371/journal.pone.0068910

Yan, C., and Zang, Y. (2010). DPARSF: a MATLAB toolbox for "pipeline" data analysis of resting-state fMRI. Front. Syst. Neurosci. 4:13. doi: 10.3389/fnsys.2010.00013

Zalesky, A., Fornito, A., Cocchi, L., Gollo, L. L., and Breakspear, M. (2014). Time-resolved resting-state brain networks. Proc. Natl. Acad. Sci. U.S.A. 111, 10341-10346. doi: 10.1073/pnas.1400181111

Zalesky, A., Fornito, A., Harding, I. H., Cocchi, L., Yücel, M., Pantelis, C., et al. (2010). Whole-brain anatomical networks: does the choice of nodes matter? Neuroimage 50, 970-983. doi: 10.1016/j.neuroimage.2009.12.027

Zalesky, A., Fornito, A., Seal, M. L., Cocchi, L., Westin, C.-F., Bullmore, E. T., et al. (2011). Disrupted axonal fiber connectivity in schizophrenia. Biol. Psychiatry 69, 80-89. doi: 10.1016/j.biopsych.2010.08.022

Zar, J. H. (1996). Biostatistical Analysis. Upper Saddle River, NJ: Prentice Hall.

Zuo, X., Xu, T., Jiang, L., Yang, Z., Cao, X., He, Y., et al. (2013). Toward reliable characterization of functional homogeneity in the human brain: preprocessing, scan duration, imaging resolution and computational space. Neuroimage 65, 374-386. doi: 10.1016/j.neuroimage.2012.10.017

Conflict of Interest Statement: The authors declare that the research was conducted in the absence of any commercial or financial relationships that could be construed as a potential conflict of interest.

Copyright (c) 2015 Liao, Yuan, Zhao, Dai, Shu, Xia, Yang, Evans and He. This is an open-access article distributed under the terms of the Creative Commons Attribution License (CC BY). The use, distribution or reproduction in other forums is permitted, provided the original author(s) or licensor are credited and that the original publication in this journal is cited, in accordance with accepted academic practice. No use, distribution or reproduction is permitted which does not comply with these terms. 